\title{
Optimal Investment-Consumption Strategy under Inflation in a Markovian Regime-Switching Market
}

\author{
Huiling Wu \\ China Institute for Actuarial Science, Central University of Finance and Economics, Beijing 100081, China \\ Correspondence should be addressed to Huiling Wu; sunnyling168@hotmail.com
}

Received 30 December 2015; Accepted 9 June 2016

Academic Editor: Silvia Romanelli

Copyright (C) 2016 Huiling Wu. This is an open access article distributed under the Creative Commons Attribution License, which permits unrestricted use, distribution, and reproduction in any medium, provided the original work is properly cited.

\begin{abstract}
This paper studies an investment-consumption problem under inflation. The consumption price level, the prices of the available assets, and the coefficient of the power utility are assumed to be sensitive to the states of underlying economy modulated by a continuous-time Markovian chain. The definition of admissible strategies and the verification theory corresponding to this stochastic control problem are presented. The analytical expression of the optimal investment strategy is derived. The existence, boundedness, and feasibility of the optimal consumption are proven. Finally, we analyze in detail by mathematical and numerical analysis how the risk aversion, the correlation coefficient between the inflation and the stock price, the inflation parameters, and the coefficient of utility affect the optimal investment and consumption strategy.
\end{abstract}

\section{Introduction}

The investment-consumption optimization problem has been one of the time-honored topics in which the decision-maker seeks to maximize the expected utility of intertemporal consumption plus the terminal wealth. The classical investmentconsumption model can be traced back to the foundational work of Samuelson [1], Hakansson [2], Fama [3], and Merton [4]. Their foundational work has inspired various extensions and applications from different aspects in the past forty years, including Zariphopoulou [5], Akian et al. [6], Liu [7], Zhao and Nie [8], and Dai et al. [9] with transaction costs, Taksar and Sethi [10] and Zariphopoulou [11] with bankruptcy, Munk and Sørensen [12] and Wang and Yi [13] with stochastic interest rate or stochastic return rate, Munk [14] and Dybvig and Liu [15] with a stochastic income, and Pliska and Ye [16] and Kwak et al. [17] with a life insurance purchase.

No matter what the models are, the existing research papers mentioned above share the common setting that the investor makes the investment-consumption decision under the uncertainty of assets' prices. However, the uncertainty of the assets' prices also comes from the uncertainty of underlying economy. To be more specific, the market mode in a real world usually has a finite number of states, such as "bullish" and "bearish" and could switch among them. It is called "regime switching." The empirical analysis shows that the returns of the assets, such as the stocks' appreciation rates and volatility rates, are sensitive to the states of underlying economy and are quite different in different states. For example, Hardy [18] used monthly data from the Toronto Stock Exchange 300 indices and the Standard and Poor's 500 to fit a regime-switching log-normal model and found that the regime-switching model is better than all the models considered. Usually the movement of the market states is depicted by a continuous-time finite-state Markovian chain and the asset's return at time $t$ is assumed to be a function of the current market state. In the past years, there has been great interest of using regime-switching models in finance and actuarial science. Here we review the related literature with regime switching according to the research topic. Zhou and Yin [19], Çakmak and Özekici [20], Celikyurt and Özekici [21], Wei and $\mathrm{Ye}[22], \mathrm{Wu}$ and $\mathrm{Li}[23,24]$, and $\mathrm{Wu}$ and Zeng [25] considered the optimal investment strategy under the meanvariance criterion while Chen et al. [26] investigated an asset-liability management problem. Cheung and Yang [27] and Çanakoğlu and Ozekici $[28,29]$ studied the investment policy under the power utility but the latter ones assumed that the utilities have the regime-dependent parameters; 
that is, the utility is being of the form $U(i, x)=K(i)+$ $C(i)(x-\beta)^{\gamma} / \gamma$. As for the optimal investment-consumption problem, Cheung and Yang [30] considered a multiperiod model where the return of the risky assets depends on the economic environments with an absorbing state which represents the bankruptcy state. Li et al. [31] and Zeng et al. [32] investigated a discrete-time investment-consumption problem with regime switching and uncertain time horizon. Gassiat et al. [33] studied this problem in an illiquid financial market where the asset trading has time restriction. Pirvu and Zhang [34] considered a continuous-time investmentconsumption problem with regime-switching discount rate and asset returns. In this paper, enlightened by the existing literature, we assume that the utility function is of this form $U(x)=\zeta(i) x^{\gamma} / \gamma$, where $\zeta(i)$ is dependent on the current market state $i$. Under this assumption, the utility function is changed according to the market states over time. In this sense, our paper has adopted the similar assumption about the movement of the financial market and the utility parameters as some existing literature. For example, Çanakoğlu and Ozekici $[28,29]$ also assumed that the parameters of the power utility are state-dependent. However, there are some differences between our paper and the existing literature. Firstly, the optimization problem considered is different. Çanakoğlu and Ozekici $[28,29]$ investigated an optimal portfolio selection problem while our paper studies an investment-consumption strategy. Secondly, the abovementioned research papers do not analyze the effects of the inflation. In contrast, in addition to the financial risk, the inflation risk is also considered in our paper.

In recent years, the phenomenon of inflation has been causing grave concern in developed and developing countries. When the continuous increase in price level exceeds a tolerable limit, the inflation can cause many distortions in investment behavior and effect greatly on daily life of the people. The persistence of inflation can diminish the investment enthusiasm on the normal financial products since the investors are not really earning money. They prefer acquisition of land and other assets which yield quick capital gains. When inflation continues over a period of time, it also erodes the motivation for saving due to the fact that the money is worth more presently than in the future. For example, if the return of the bank account was $4 \%$ and the inflation was $5 \%$, then the real return on investment would be minus $1 \%$. In addition, when commodity price is raised, the consumers cannot buy as much as they could previously, and hence they have opted for major cuts in their daily budget. Nowadays, the problem of inflation is quite common for the people all over the world. Therefore, we think that it is theoretically and practically important to consider the investment-consumption problem under inflation.

However, none of the above papers allow for stochastic inflation. Here, we introduce some existing literature allowing for inflation. For the optimal portfolio selection problem under inflation, Brennan and Xia [35], Munk et al. [36], and Chiarella et al. [37] aimed to maximize the expected power utility from terminal real wealth and obtained the closedform investment strategies for the investors. Menoncin [38] studied an optimal portfolio selection problem for a HARA utility investor under stochastic inflation and wage income. He obtained a quasiexplicit solution for this problem. Mamun and Visaltanachoti [39] analyzed numerically how the anticipated rate of inflation affected the investment strategy of US investors under the assumption that the assets available included treasury inflation protected securities, equity, real estate, treasury bonds, and corporate bonds. Their study indicated that when the anticipated rate of inflation is higher, the investor should allocate more wealth to the treasury inflation protected securities. For the defined contribution management problem under inflation, Battocchio and Menoncin [40] considered an optimal pension management under stochastic interest rate, wage income, and inflation. They wanted to maximize the expected exponential utility from terminal wealth and found a closed-form solution for this problem. Zhang and Ewald [41] assumed that the financial market consists of a money account, a stock, and an inflation linked bond. They wanted to maximize the expected power utility from the terminal wealth and obtained the optimal investment strategy using the martingale method. Han and Hung [42] assumed that the retired individual received a guarantee as a downside protection. The closedform solution is obtained under the power utility function. For more information, refer to Battocchio and Menoncin [43], Zhang et al. [44], and de Jong [45]. For the optimal consumption problem under inflation, Brennan and Xia [35] investigated a problem for the interim consumption under the power utility and obtained the explicit expression of the consumption. Menoncin [46] generalized Menoncin [38] to the case with intertemporal consumption. He aimed to maximize the expected HARA utility of the intertemporal consumption plus the terminal wealth under the stochastic income and inflation and computed a quasiexplicit solution for both optimal consumption and investment. Chou et al. [47] considered an optimal portfolio-consumption problem under stochastic inflation with nominal and indexed bonds. They studied, respectively, an optimization problem that aims to maximize the expected terminal wealth at a fixed terminal time $T$ and an optimization problem that maximizes the intertemporal consumption utility with infinite time horizon. Paradiso et al. [48] studied the existence and stability of the consumption function in the United States of America since the 1950s. They introduced inflation as an additional explanatory variable to analyze the life-cycle consumption function.

We can see that literature on optimal investment-consumption under inflation is so limited. Moreover, the existing literature has not studied how the commodity price level affects the optimal investment-consumption decision of the investors in a Markovian regime-switching market as mentioned above. This paper aims to bridge the gap. Referring to Korn et al. [49], we assume that the instantaneous expected rate and volatility rate of inflation are also dependent on the market states.

The rest of our paper is organized as follows. The problem formulation and the verification theory are presented in Section 2. The explicit expressions of the investment strategy and consumption are obtained in Section 3. The properties of investment strategy are analyzed mathematically in Section 4. 
The properties of the optimal consumption proportion are demonstrated in Section 5 by mathematical and numerical analysis. This paper is concluded in Section 6.

\section{Problem Formulation and Notations}

In this paper, there are a bank account and a stock traded continuously within a time horizon $[0, T]$ whose price processes depend on the states of an underlying economy. Here the evolution of the market states is modulated by a continuoustime Markov chain $\{\xi(t): t \geq 0\}$ taking discrete values in a finite space $S=\{1,2, \ldots, L\}$ and having a generator $Q=\left(q_{i j}\right)_{i, j \in S}$. The price process of the bank account satisfies the following differential equation:

$$
d A_{0}(t)=A_{0}(t) r(t, \xi(t)) d t, \quad A(0)=a_{0},
$$

where $r(t, \xi(t))$ is the instantaneous interest rate of the bank account corresponding to the market state $\xi(t)$. The evolution of the price process of the stock is governed by the following Markovian regime-switching geometric Brownian motion:

$$
\begin{array}{r}
d A_{1}(t)=A_{1}(t)[\mu(t, \xi(t)) d t+\sigma(t, \xi(t)) d W(t)], \\
A_{1}(0)=a_{1},
\end{array}
$$

where $W(t)$ is a standard one-dimensional Brownian motion and $\mu(t, \xi(t))$ and $\sigma(t, \xi(t))$ are, respectively, the appreciation rate and volatility rate of the stock corresponding to the market state $\xi(t)$.

Let $I(t)$ denote the nominal price level per unit of consumption goods at time $t$. Then, the evolution of $I(t)$ is assumed to follow the stochastic differential equation:

$$
\begin{array}{r}
d I(t)=I(t)\left[\mu_{I}(t, \xi(t)) d t+\sigma_{I}(t, \xi(t)) d W_{I}(t)\right] \\
I(0)=a_{2}>0,
\end{array}
$$

where $W_{I}(t)$ is a standard one-dimensional Brownian motion and $\mu_{I}(t, \xi(t))$ and $\sigma_{I}(t, \xi(t))$ are the expected inflation rate and volatility rate at time $t$, respectively. Generally, we assume that $W(t)$ and $W_{I}(t)$ are correlated with a correlation coefficient $\rho(t) \in[-1,1]$. Referring to Koo [50], (3) can be expressed as

$$
\begin{aligned}
& d I(t)=I(t)\left[\mu_{I}(t, \xi(t)) d t+\sigma_{I}(t, \xi(t)) \rho(t) d W(t)\right. \\
& \left.+\sigma_{I}(t, \xi(t)) \sqrt{1-\rho^{2}(t)} d W_{0}(t)\right]
\end{aligned}
$$

where $W_{0}(t)$ is a standard one-dimensional Brownian motion independent of $W(t)$. Furthermore, we assume that $\xi(t)$ and $\left(W_{0}(t), W(t)\right)$ are independent of each other. To describe uncertainty, we employ a complete filtered probability space $\left(\Omega, \mathscr{F}, P,\left\{\mathscr{F}_{t}\right\}_{t \geq 0}\right)$, where $\mathscr{F}_{t}$ is defined as $\mathscr{F}_{t}=$ $\sigma\left\{\left(W_{0}(s), W(s)\right), \xi(s): 0 \leq s \leq t\right\}$. We also assume throughout this paper that $r(t, i), \mu(t, i), \sigma(t, i), \mu_{I}(t, i)$, and $\sigma_{I}(t, i)$ are deterministic and uniformly bounded in $t$ for any given state $\xi(t)=i$.

Referring to Menoncin [46], the variable $I^{-1}(t)$ "represents the purchasing power of a nominal monetary unit. Furthermore, if we identify the value of a monetary unit with the number of goods that can be purchased against it, then $I^{-1}(t)$ can also be interpreted as the value of money."

An investor joins the market at time 0 with initial wealth $x_{0}$ and plans to invest and consume his wealth dynamically over a fixed time horizon $T$. Let $\theta(t)$ be the proportion of the wealth available invested in the stock at time $t$ and let $c(t) \geq 0$ be the real consumption, that is, the ratio between the nominal consumption and the price level $I(t)$. Then, the nominal wealth process $\left\{X^{\theta, c}(t): t \in[0, T]\right\}$ satisfies the following stochastic differential equation:

$$
\begin{aligned}
d X^{\theta, c}(t)= & X^{\theta, c}(t)(1-\theta(t)) r(t, i) d t \\
& +X^{\theta, c}(t) \theta(t)[\mu(t, i) d t+\sigma(t, i) d W(t)] \\
& -c(t) I(t) d t \\
= & X^{\theta, c}(t) r(t, i) d t \\
& +X^{\theta, c}(t) \theta(t)[\widetilde{\mu}(t, i) d t+\sigma(t, i) d W(t)] \\
& -c(t) I(t) d t,
\end{aligned}
$$

where $\tilde{\mu}(t, i)=\mu(t, i)-r(t, i)$.

Denote by $\bar{X}^{\theta, c}(t)=X^{\theta, c}(t) / I(t)$ the real wealth level at time $t$ after considering the inflation. Then according to Itô's formula, the dynamics of $\bar{X}^{\theta, c}(t)$ is

$$
\begin{aligned}
& d \bar{X}^{\theta, c}(t)=d\left(\frac{X^{\theta, c}(t)}{I(t)}\right)=\bar{X}^{\theta, c}(t)\left[r(t, i)-\mu_{I}(t, i)\right. \\
& +\left(\sigma_{I}(t, i)\right)^{2} \\
& \left.+\theta(t)\left(\widetilde{\mu}(t, i)-\rho(t) \sigma(t, i) \sigma_{I}(t, i)\right)\right] d t \\
& -c(t) d t+\bar{X}^{\theta, c}(t) \theta(t) \sigma(t, i) d W(t)-\bar{X}^{\theta, c}(t) \\
& \cdot \sigma_{I}(t, i)\left(\rho(t) d W(t)+\sqrt{1-\rho^{2}(t)} d W_{0}(t)\right)
\end{aligned}
$$

with initial value $\bar{X}(0)=\bar{x}_{0}=x_{0} / a_{2}$.

The investor's optimization problem could be described by the following:

$$
\max _{\mathbb{A}(0, T)} \mathrm{E}_{0, i_{0}, \bar{x}_{0}}\left[\int_{0}^{T} e^{-\delta t} U(\xi(t), c(t)) d t+e^{-\delta T} U\left(\xi(T), \bar{X}^{\theta, c}(T)\right)\right],
$$


where $\delta$ is the discount rate and the set of admissible strategies $\mathbb{A}(0, T)$ is defined below. In our paper, the utility function $U(i, x)$ is defined as $U(i, x)=\zeta(i) x^{\gamma} / \gamma$, where $\gamma<1, \gamma \neq 0$, and $\zeta(i)>0$.

Definition 1. A strategy $\{(\theta(t), c(t) \geq 0): 0 \leq t \leq T\}$ is admissible if

(i) for any initial wealth $\bar{x}_{0}>0$ the stochastic differential equation (6) has a unique solution $\bar{X}^{\theta, c}(t)$ corresponding to $(\theta(t), c(t))$;

(ii) the corresponding solution $\bar{X}^{\theta, c}(\cdot)$ satisfies $\mathrm{E}\left(\sup _{t \in[0, T]}\left|\bar{X}^{\theta, c}(t)\right|^{2 \gamma}\right)<+\infty$ for all $\gamma \leq 1$;

(iii) $\mathrm{E}\left(\int_{0}^{T}(\theta(t))^{2} d t\right)<+\infty, \mathrm{E}\left(\int_{0}^{T}(c(t))^{\gamma} d t\right)<+\infty$ for all $\gamma \leq 1$

(iv) $\bar{X}^{\theta, c}(T)>0$ a.s.

For convenience, denote by $\mathbb{A}(t, T)$ the set of admissible strategies $\{(\theta(s), c(s)): t \leq s \leq T\}$.

We can write the value function in $t \in[0, T)$ as

$$
\begin{aligned}
& V(t, x, i)=\max _{\mathbb{A}(t, T)} \mathrm{E}_{t, i, x}\left[\int_{t}^{T} e^{-\delta(s-t)} U(\xi(s), c(s)) d s\right. \\
& \left.+e^{-\delta(T-t)} U\left(\xi(T), \bar{X}^{\theta, c}(T)\right)\right]
\end{aligned}
$$

with terminal condition $V(T, x, i)=U(i, x)$.

Then, the optimal investment-consumption problem can be formulated by the dynamic programming equation

$$
\begin{aligned}
- & \delta V(t, x, i)+V_{t}(t, x, i)+\sum_{j=1}^{L} q_{i j} V(t, x, j)+\frac{1}{2} \\
\cdot & x^{2} V_{x x}(t, x, i)\left(\sigma_{I}(t, i)\right)^{2}+\sup _{\theta(t), c(t) \geq 0}\{U(i, c(t)) \\
+ & V_{x}(t, x, i)[x(\eta(t, i)+\theta(t) \kappa(t, i))-c(t)]+\frac{1}{2} \\
\cdot & x^{2} V_{x x}(t, x, i) \\
\cdot & {\left.\left[\theta^{2}(t) \sigma^{2}(t, i)-2 \theta(t) \rho(t) \sigma(t, i) \sigma_{I}(t, i)\right]\right\}=0, }
\end{aligned}
$$

where $\eta(t, i)=r(t, i)-\mu_{I}(t, i)+\left(\sigma_{I}(t, i)\right)^{2}$ and $\kappa(t, i)=\widetilde{\mu}(t, i)-$ $\rho(t) \sigma(t, i) \sigma_{I}(t, i)$.

The optimality condition (9) is not sufficient if a verification theorem is not provided, so we present the verification theorem before we give the explicit solution to this problem. Let $C^{1,2}([0, T] \times \mathrm{O} \times S)$, where $\mathrm{O} \subseteq \mathbb{R}$ denote the set of all continuous functions $f(t, x, i):[0, T] \times \mathrm{O} \times S \rightarrow \mathbb{R}$ that are continuously differentiable in $t$ and twice continuously differentiable in $x$ for any $i \in S$.
Theorem 2. Let $v(t, x, i) \in C^{1,2}([0, T] \times O \times S)$, where $O \subseteq \mathbb{R}$, be a solution to the HJB equation (9) with boundary condition $V(T, x, i)=U(i, x)$. If for all $(t, x, i) \in[0, T] \times O \times S$ and all admissible controls there exists $\beta>1$ such that

$$
\mathrm{E}_{t, i, x}\left(\sup _{s \in[t, T]}\left|v\left(s, \bar{X}^{\theta, c}(s), \xi(s)\right)\right|^{\beta}\right)<+\infty,
$$

then we have

(a) $v(t, x, i) \geq V(t, x, i)$;

(b) if there exists an admissible strategy $\left(\theta^{*}(\cdot), c^{*}(\cdot)\right)$ that is a maximizer of (9), then $v(t, x, i)=V(t, x, i)$ for all $i \in S, x \in O$, and $t \in[0, T]$. Furthermore, $\left(\theta^{*}(\cdot), c^{*}(\cdot)\right)$ is an optimal strategy.

Proof. (a) Applying Itô's formula to $e^{\delta(T-t)} v(t, x, i)$ yields

$$
\begin{aligned}
& U\left(\xi(T), \bar{X}^{\theta, c}(T)\right)=v\left(T, \bar{X}^{\theta, c}(T), \xi(T)\right) \\
& =e^{\delta(T-t)} v(t, x, i) \\
& +\int_{t}^{T}-\delta e^{\delta(T-s)} v\left(s, \bar{X}^{\theta, c}(s), \xi(s)\right) d s \\
& +\int_{t}^{T} e^{\delta(T-s)} v_{t}\left(s, \bar{X}^{\theta, c}(s), \xi(s)\right) d s+\int_{t}^{T} e^{\delta(T-s)} \\
& \cdot v_{x}\left(s, \bar{X}^{\theta, c}(s), \xi(s)\right) \\
& \cdot\left[\bar{X}^{\theta, c}(s)(\eta(s, \xi(s))+\theta(s) \kappa(s, \xi(s)))-c(s)\right] d s \\
& +\frac{1}{2} \int_{t}^{T} e^{\delta(T-s)}\left(\bar{X}^{\theta, c}(s)\right)^{2} v_{x x}\left(s, \bar{X}^{\theta, c}(s), \xi(s)\right) \\
& \times\left[\left(\theta(s) \sigma(s, \xi(s))-\sigma_{I}(s, \xi(s)) \rho(s)\right)^{2}\right. \\
& \left.+\left(\sigma_{I}(s, \xi(s))\right)^{2}\left(1-\rho^{2}(s)\right)\right] d s+\int_{t}^{T} e^{\delta(T-s)} \\
& \cdot \sum_{j=1}^{L} q_{\xi(s) j} v\left(s, \bar{X}^{\theta, c}(s), j\right) d s+\int_{t}^{T} e^{\delta(T-s)} \\
& \cdot v_{x}\left(s, \bar{X}^{\theta, c}(s), \xi(s)\right) \bar{X}^{\theta, c}(s)[\theta(s) \sigma(s, \xi(s)) \\
& \left.-\sigma_{I}(s, \xi(s)) \rho(s)\right] d W(s)-\int_{t}^{T} e^{\delta(T-s)} \\
& \cdot v_{x}\left(s, \bar{X}^{\theta, c}(s), \xi(s)\right) \bar{X}^{\theta, c}(s) \sigma_{I}(s, \xi(s)) \\
& \cdot \sqrt{1-\rho^{2}(s)} d W_{0}(s) \text {. }
\end{aligned}
$$




\section{Denote}

$$
\begin{aligned}
& \mathrm{A}^{\theta, c} v(t, x, i)=U(i, c(t))-\delta v(t, x, i)+v_{t}(t, x, i) \\
& +\sum_{j=1}^{L} q_{i j} v(t, x, j)+\frac{1}{2} x^{2} v_{x x}(t, x, i)\left(\sigma_{I}(t, i)\right)^{2} \\
& +v_{x}(t, x, i)[x(\eta(t, i)+\theta(t) \kappa(t, i))-c(t)]+\frac{1}{2} \\
& \cdot x^{2} v_{x x}(t, x, i) \\
& \cdot\left[(\theta(t))^{2}(\sigma(t, i))^{2}-2 \theta(t) \rho \sigma(t, i) \sigma_{I}(t, i)\right] .
\end{aligned}
$$

Thus, we have

$$
\begin{aligned}
& \int_{t}^{T} e^{-\delta(s-t)} U(\xi(s), c(s)) d s+e^{-\delta(T-t)} U(\xi(T), \\
& \left.\bar{X}^{\theta, c}(T)\right)=v(t, x, i) \\
& +\int_{t}^{T} e^{-\delta(s-t)} A^{\theta, c} v\left(s, \bar{X}^{\theta, c}(s), \xi(s)\right) d s \\
& +\int_{t}^{T} e^{-\delta(s-t)} v_{x}\left(s, \bar{X}^{\theta, c}(s), \xi(s)\right) \bar{X}^{\theta, c}(s) \\
& \cdot[\theta(s) \sigma(s, \xi(s)) \\
& \left.-\sigma_{I}(s, \xi(s)) \rho(s)\right] d W(s) \\
& -\int_{t}^{T} e^{-\delta(s-t)} v_{x}\left(s, \bar{X}^{\theta, c}(s), \xi(s)\right) \bar{X}^{\theta, c}(s) \sigma_{I}(s, \xi(s)) \\
& \cdot \sqrt{1-\rho^{2}(s)} d W_{0}(s) .
\end{aligned}
$$

We first assume that $\mathrm{O} \in \mathbb{R}$ is bounded. When $v(t, x, i) \in$ $C^{1,2}([0, T] \times \mathrm{O} \times S)$ and $\theta(t)$ and $c(t)$ are admissible, according to Definition 1 , we know that

$$
\begin{aligned}
& \int_{t}^{T} e^{-\delta(s-t)} v_{x}\left(s, \bar{X}^{\theta, c}(s), \xi(s)\right) \bar{X}^{\theta, c}(s)[\theta(s) \sigma(s, \xi(s)) \\
& \left.-\sigma_{I}(s, \xi(s)) \rho(s)\right] d W(s) \\
& \int_{t}^{T} e^{-\delta(s-t)} v_{x}\left(s, \bar{X}^{\theta, c}(s), \xi(s)\right) \bar{X}^{\theta, c}(s) \sigma_{I}(s, \xi(s)) \\
& \cdot \sqrt{1-\rho^{2}(s)} d W_{0}(s)
\end{aligned}
$$

are martingales, and $\mathrm{E}\left[\int_{t}^{T} e^{-\delta(s-t)} A^{\theta, c} v\left(s, \bar{X}^{\theta, c}(s), \xi(s)\right) d s\right]<$ $+\infty$. Since $v(t, x, i)$ solves HJB equation (9), taking expectation on both sides of the above equality yields

$$
\begin{aligned}
& \mathrm{E}\left[\int_{t}^{T} e^{-\delta(s-t)} U(\xi(s), c(s)) d s\right. \\
& \left.\quad+e^{-\delta(T-t)} U\left(\xi(T), \bar{X}^{\theta, c}(T)\right)\right] \leq v(t, x, i),
\end{aligned}
$$

In the general case when $\mathrm{O} \in \mathbb{R}$ might not be bounded, for a relatively fixed time $t \in[0, T)$, we define

$$
\begin{aligned}
\mathrm{O}_{p}= & \mathrm{O} \\
& \cap\left\{z \in \mathbb{R}:|z|<p, \operatorname{dist}(z, \partial \mathrm{O})>p^{-1}, p \in \mathbb{N}\right\}, \\
Q_{p}= & {\left[t, T-p^{-1}\right) \times \mathrm{O}_{p}, }
\end{aligned}
$$

where $p$ satisfies $p^{-1}<T$ and $T-p^{-1}>t$. Let $\tau_{p}$ be the first exit time of stochastic process $\left(s, \bar{X}^{\theta, c}(s)\right)_{s \geq t}$ from $Q_{p}$ and $\omega_{p}=\min \left\{\tau_{p}, T\right\}$. Then, $\left\{\omega_{p}, p \in \mathbb{N}\right\}$ is a sequence of stopping times. Furthermore, as $p \rightarrow+\infty, \omega_{p}$ increases to $T$ with probability 1 . Since now $O_{p}$ is bounded, referring to the analysis above, we can derive

$$
\begin{aligned}
& \mathrm{E}\left[\int_{t}^{\omega_{p}} e^{-\delta(s-t)} U(\xi(s), c(s)) d s\right. \\
& \left.\quad+e^{-\delta\left(\omega_{p}-t\right)} v\left(\omega_{p}, \bar{X}^{\theta, c}\left(\omega_{p}\right), \xi\left(\omega_{p}\right)\right)\right] \leq v(t, x, i) .
\end{aligned}
$$

Equation (10) implies uniform integrability of $v(t, x, i)$. Therefore, we have

$$
\begin{aligned}
& v(t, x, i) \geq \lim _{p \rightarrow+\infty} \mathrm{E}\left[\int_{t}^{\Phi_{p}} e^{-\delta(s-t)} U(\xi(s), c(s)) d s\right. \\
& \left.+e^{-\delta\left(\varrho_{p}-t\right)} v\left(\omega_{p}, \bar{X}^{\theta, c}\left(\omega_{p}\right), \xi\left(\omega_{p}\right)\right)\right] \\
& =\mathrm{E}\left[\int_{t}^{T} e^{-\delta(s-t)} U(\xi(s), c(s)) d s\right. \\
& \left.+e^{-\delta(T-t)} U\left(\xi(T), \bar{X}^{\theta, c}(T)\right)\right]
\end{aligned}
$$

which implies that $v(t, x, i) \geq V(t, x, i)$.

(b) When taking the strategy $\left\{\left(\theta^{*}(t), c^{*}(t)\right): 0 \leq t \leq\right.$ $T\}$, the inequalities become equalities. Hence, conclusion (b) holds.

\section{Optimal Investment-Consumption Strategy}

In this section, we assume that the utility of the investor in state $i$ is given by the power utility function

$$
U(i, x)=\zeta(i) \frac{x^{\gamma}}{\gamma},
$$

where $\zeta(i)>0$ for all $i \in S, x>0, \gamma<1$, and $\gamma \neq 0$.

Suppose that a solution to HJB equation (9) is of this form:

$$
\begin{aligned}
& v(t, x, i)=\bar{\zeta}(t, i) \frac{x^{\gamma}}{\gamma}, \\
& v(T, x, i)=\zeta(i) \frac{x^{\gamma}}{\gamma} .
\end{aligned}
$$


Then, substituting (20) into (9) yields

$$
\begin{aligned}
& -\delta \bar{\zeta}(t, i) \frac{x^{\gamma}}{\gamma}+\bar{\zeta}_{t}(t, i) \frac{x^{\gamma}}{\gamma}-\frac{1}{2} \bar{\zeta}(t, i)(1-\gamma) \\
& -x^{\gamma}\left(\sigma_{I}(t, i)\right)^{2}+\bar{\zeta}(t, i) \eta(t, i) x^{\gamma}+\frac{x^{\gamma}}{\gamma} \sum_{j=1}^{L} q_{i j} \bar{\zeta}(t, j) \\
& +\sup _{\theta(t), c(t) \geq 0}\left\{\zeta(i) \frac{c(t)^{\gamma}}{\gamma}-\bar{\zeta}(t, i) x^{\gamma-1} c(t)\right. \\
& +\bar{\zeta}(t, i) x^{\gamma} \theta(t) \kappa(t, i) \\
& -\frac{1}{2} \bar{\zeta}(t, i)(1-\gamma) x^{\gamma} \theta^{2}(t) \sigma^{2}(t, i) \\
& \left.+\bar{\zeta}(t, i)(1-\gamma) x^{\gamma} \theta(t) \rho(t) \sigma(t, i) \sigma_{I}(t, i)\right\}=0
\end{aligned}
$$

where $\bar{\zeta}_{t}(t, i)$ is the partial derivative to $t$.

If $\bar{\zeta}(t, i)>0$ and $x>0$, differentiating with respect to $\theta(t)$ and $c(t)$ in (21), respectively, gives the maximizers as follows:

$$
\begin{aligned}
\theta^{*}(t, i) & =\frac{\kappa(t, i)+(1-\gamma) \rho(t) \sigma(t, i) \sigma_{I}(t, i)}{(1-\gamma) \sigma^{2}(t, i)}, \\
c^{*}(t, x, i) & =\left(\frac{\zeta(i)}{\bar{\zeta}(t, i)}\right)^{1 /(1-\gamma)} x,
\end{aligned}
$$

where $\bar{\zeta}(t, i)$ solves the following equation:

$$
\begin{aligned}
& 0= \bar{\zeta}_{t}(t, i)+(1-\gamma) \zeta(i)\left(\frac{\zeta(i)}{\bar{\zeta}(t, i)}\right)^{\gamma /(1-\gamma)} \\
&+\sum_{j=1}^{L} q_{i j} \bar{\zeta}(t, j)+\bar{\zeta}(t, i)(\gamma \eta(t, i)-\delta \\
&+\frac{1}{2} \frac{\gamma}{1-\gamma} \frac{\kappa^{2}(t, i)}{\sigma^{2}(t, i)}+\gamma \frac{\rho(t) \kappa(t, i) \sigma_{I}(t, i)}{\sigma(t, i)} \\
&\left.-\frac{1}{2} \gamma(1-\gamma)\left(1-\rho^{2}(t)\right)\left(\sigma_{I}(t, i)\right)^{2}\right), \\
& \bar{\zeta}(T, i)=\zeta(i)>0 .
\end{aligned}
$$

Next we shall show that $\bar{\zeta}(t, i)>0$ and the wealth process $\bar{X}^{\theta^{*}, c^{*}}(t)>0$ by the following lemmas step by step.

Lemma 3. If $\bar{\zeta}(t, i)$ solves (24), then

(a) $\bar{\zeta}(t, i)>0$; furthermore, $\bar{\zeta}(t, i)$ is uniformly bounded from below; that is, there exists a constant $\widetilde{M}>0$ such that $\bar{\zeta}(t, i) \geq \widetilde{M}$;

(b) $\bar{\zeta}(t, i)$ is the only continuous solution of $(24)$, and $\bar{\zeta}(t, i)$ has an uniformly upper bound in $[0, T] \times S$.
Proof. (a) Denote

$$
\begin{aligned}
& \phi(t, i)=\gamma \eta(t, i)-\delta+\frac{1}{2} \frac{\gamma}{1-\gamma} \frac{\kappa^{2}(t, i)}{\sigma^{2}(t, i)} \\
& +\gamma \frac{\rho(t) \kappa(t, i) \sigma_{I}(t, i)}{\sigma(t, i)} \\
& -\frac{1}{2} \gamma(1-\gamma)\left(1-\rho^{2}(t)\right)\left(\sigma_{I}(t, i)\right)^{2} \\
& =\gamma r(t, i)-\gamma \mu_{I}(t, i)-\delta+\frac{1}{2} \frac{\gamma}{1-\gamma} \frac{\tilde{\mu}^{2}(t, i)}{\sigma^{2}(t, i)} \\
& +\frac{1}{2} \gamma(1+\gamma)\left(\sigma_{I}(t, i)\right)^{2} \\
& -\frac{\gamma^{2}}{1-\gamma} \frac{\rho(t) \tilde{\mu}(t, i) \sigma_{I}(t, i)}{\sigma(t, i)} \\
& +\frac{1}{2} \frac{\gamma^{3}}{1-\gamma} \rho^{2}(t)\left(\sigma_{I}(t, i)\right)^{2}, \\
& K(t, s)=\exp \left[\int_{t}^{s} \phi(u, \xi(u)) d u\right] \text {, } \\
& M(t, s)=\sum_{t \leq v \leq s}[\bar{\zeta}(v, \xi(v))-\bar{\zeta}(v, \xi(v-))] \\
& -\int_{t}^{s} \sum_{j=1}^{L} q_{\xi(v-) j} \bar{\zeta}(v, j) d v
\end{aligned}
$$

Then, in view of (24), we have

$$
\begin{aligned}
d[ & K(t, s) \bar{\zeta}(s, \xi(s))]=\bar{\zeta}(s, \xi(s)) K_{s}(t, s) \\
& +K(t, s) d \bar{\zeta}(s, \xi(s))=K(t, s) \\
& \cdot[\phi(s, \xi(s)) \bar{\zeta}(s, \xi(s)) d s+d \bar{\zeta}(s, \xi(s))]=K(t, s) \\
& \cdot\left[\phi(s, \xi(s)) \bar{\zeta}(s, \xi(s))+\bar{\zeta}_{s}(s, \xi(s))\right. \\
& \left.+\sum_{j=1}^{L} q_{\xi(s-) j} \bar{\zeta}(s, j)\right] d s+K(t, s)[\bar{\zeta}(s, \xi(s)) \\
& -\bar{\zeta}(s, \xi(s-))]-K(t, s) \sum_{j=1}^{L} q_{\xi(s-) j} \bar{\zeta}(s, j) d s=-(1 \\
& +\gamma(t, s) d M(t, s) . \\
& +\gamma(t, s) \zeta(\xi(s))\left(\frac{\zeta(\xi(s))}{\bar{\zeta}(s, \xi(s))}\right)^{\gamma /(1-\gamma)} d s
\end{aligned}
$$


The solution of the above equation is of this form:

$$
\begin{aligned}
& K(t, T) \zeta(\xi(T))=\bar{\zeta}(t, i)-(1-\gamma) \\
& \quad \cdot \int_{t}^{T} K(t, s) \zeta(\xi(s))\left(\frac{\zeta(\xi(s))}{\bar{\zeta}(t, \xi(s))}\right)^{\gamma /(1-\gamma)} d s \\
& \quad+\int_{t}^{T} K(t, s) d M(t, s) .
\end{aligned}
$$

It is well known that $M(t, s)$ is a martingale; then, we have

$$
\begin{aligned}
\bar{\zeta}(t, i) & =\mathrm{E}_{t, i}(\zeta(\xi(T)) K(t, T))+(1-\gamma) \\
\cdot \mathrm{E}_{t, i} & {\left[\int_{t}^{T} K(t, s) \zeta(\xi(s))\left(\frac{\zeta(s, \xi(s))}{\zeta(\xi(s))}\right)^{\gamma /(\gamma-1)} d s\right] . }
\end{aligned}
$$

To prove $\bar{\zeta}(t, i)>0$, we construct a Picard iterative sequence $\left\{\bar{\zeta}^{(k)}(t, i): k=0,1,2, \ldots\right\}$ as follows:

$$
\begin{aligned}
& \bar{\zeta}^{(0)}(t, i)=\zeta(i), \\
& \bar{\zeta}^{(k+1)}(t, i)=\mathrm{E}_{t, i}(\zeta(\xi(T)) K(t, T))+(1-\gamma) \\
& \cdot \mathrm{E}_{t, i}\left[\int_{t}^{T} K(t, s)[\zeta(\xi(s))]^{1 /(1-\gamma)}\right. \\
& \left.\cdot\left(\bar{\zeta}^{(k)}(s, \xi(s))\right)^{\gamma /(\gamma-1)} d s\right] .
\end{aligned}
$$

Noting that $\zeta(i)>0$ and $K(t, s)>0$, we have

$$
\bar{\zeta}^{(k)}(t, i) \geq \mathrm{E}_{t, i}[\zeta(\xi(T)) K(t, T)]>0, \quad k=1,2, \ldots
$$

Since all the coefficients in our paper are uniformly bounded, (32) indicates that $\bar{\zeta}^{(k)}(t, i)>\widetilde{M}>0$ for $k=1,2, \ldots$ At the same time, it is well known that $\bar{\zeta}(t, i)$ is the limit of the sequence $\left\{\bar{\zeta}^{(k)}(t, i), k=0,1,2, \ldots\right\}$ as $k \rightarrow+\infty$. Thus, $\bar{\zeta}(t, i) \geq$ $\widetilde{M}>0, t \in[0, T]$.

(b) For $i=1,2, \ldots, L$, denote

$$
\begin{aligned}
f_{i}(t, \bar{\zeta}(t, 1), \bar{\zeta}(t, 2), \ldots, \bar{\zeta}(t, L)) \\
=-(1-\gamma) \zeta(i)\left(\frac{\zeta(i)}{\bar{\zeta}(t, i)}\right)^{\gamma /(1-\gamma)}-\sum_{j=1}^{L} q_{i j} \bar{\zeta}(t, j) \\
\quad-\bar{\zeta}(t, i) \phi(t, i)
\end{aligned}
$$

We have

$$
\begin{aligned}
\bar{\zeta}_{t}(t, i)=f_{i}(t, \bar{\zeta}(t, 1), \bar{\zeta}(t, 2), \ldots, \bar{\zeta}(t, L)) & \\
& i=1,2, \ldots, L
\end{aligned}
$$

which is a system of the first-order ordinary differential equations. Since $\phi(t, i)$ is uniformly bounded for $i \in S, f_{i}$ satisfies that

$$
\begin{aligned}
& \mid f_{i}(t, \bar{\zeta}(t, 1), \bar{\zeta}(t, 2), \ldots, \bar{\zeta}(t, L)) \\
& \quad-f_{i}\left(t, \bar{\zeta}^{*}(t, 1), \bar{\zeta}^{*}(t, 2), \ldots, \bar{\zeta}^{*}(t, L)\right) \mid \\
& \quad=\mid-(1-\gamma)(\zeta(i))^{1 /(1-\gamma)} \\
& \quad \cdot\left[(\bar{\zeta}(t, i))^{\gamma /(\gamma-1)}-\left(\bar{\zeta}^{*}(t, i)\right)^{\gamma /(\gamma-1)}\right]-\sum_{j=1}^{L} q_{i j} \\
& \quad \cdot\left[\bar{\zeta}(t, j)-\bar{\zeta}^{*}(t, j)\right]-\phi(t, i)\left[\bar{\zeta}(t, i)-\bar{\zeta}^{*}(t, i)\right] \mid \\
& \quad \leq A_{1}\left|(\bar{\zeta}(t, i))^{\gamma /(\gamma-1)}-\left(\bar{\zeta}^{*}(t, i)\right)^{\gamma /(\gamma-1)}\right| \\
& \quad+A_{2} \sum_{j=1}^{L}\left|\bar{\zeta}(t, j)-\bar{\zeta}^{*}(t, j)\right|
\end{aligned}
$$

for suitable constants $A_{1}$ and $A_{2}$. Moreover,

$$
\begin{aligned}
\left|\frac{\partial(\bar{\zeta}(t, i))^{\gamma /(\gamma-1)}}{\partial \bar{\zeta}(t, i)}\right| & =\left|\frac{\gamma}{1-\gamma}\right|\left(\frac{1}{\bar{\zeta}(t, i)}\right)^{1 /(1-\gamma)} \\
& \leq\left|\frac{\gamma}{1-\gamma}\right|\left(\frac{1}{\widetilde{M}}\right)^{1 /(1-\gamma)} .
\end{aligned}
$$

Then,

$$
\begin{aligned}
& \left|(\bar{\zeta}(t, i))^{\gamma /(\gamma-1)}-\left(\bar{\zeta}^{*}(t, i)\right)^{\gamma /(\gamma-1)}\right| \\
& \quad \leq\left|\frac{\gamma}{1-\gamma}\right|\left(\frac{1}{\widetilde{M}}\right)^{1 /(1-\gamma)}\left|\bar{\zeta}(t, i)-\bar{\zeta}^{*}(t, i)\right| .
\end{aligned}
$$

Therefore,

$$
\begin{aligned}
& \mid f_{i}(t, \bar{\zeta}(t, 1), \bar{\zeta}(t, 2), \ldots, \bar{\zeta}(t, L)) \\
& \quad-f_{i}\left(t, \bar{\zeta}^{*}(t, 1), \bar{\zeta}^{*}(t, 2), \ldots, \bar{\zeta}^{*}(t, L)\right) \mid \\
& \quad \leq A_{3} \sum_{j=1}^{L}\left|\bar{\zeta}(t, j)-\bar{\zeta}^{*}(t, j)\right|,
\end{aligned}
$$

which leads to

$$
\begin{aligned}
& \sum_{i=1}^{L} \mid f_{i}(t, \bar{\zeta}(t, 1), \bar{\zeta}(t, 2), \ldots, \bar{\zeta}(t, L)) \\
& \quad-f_{i}\left(t, \bar{\zeta}^{*}(t, 1), \bar{\zeta}^{*}(t, 2), \ldots, \bar{\zeta}^{*}(t, L)\right) \mid \\
& \quad \leq A_{4} \sum_{j=1}^{L}\left|\bar{\zeta}(t, j)-\bar{\zeta}^{*}(t, j)\right| .
\end{aligned}
$$

Now it obvious that $f_{i}$ 's satisfy Lipschitz condition. Consequently, (24) has a unique continuous solution denoted by 
$\bar{\zeta}(t, i)$ in $[0, T]$. A continuous function $\bar{\zeta}(t, i)$ defined in a close interval $[0, T]$ must have an upper bound $\bar{M}_{i}$. If we define $\bar{M}=\max \left\{\bar{M}_{1}, \bar{M}_{2}, \ldots, \bar{M}_{L}\right\}$, we know that $\bar{\zeta}(t, i)$ has a uniformly upper bound $\bar{M}$.

The next step is to prove that the stochastic differential equation (6) under $\theta^{*}(t, i)$ in (22) and $c^{*}(t, x, i)$ in (23) has a unique and nonnegative solution $\bar{X}^{\theta^{*}, c^{*}}(t)$. The main results are presented in the following lemma.

Lemma 4. For any initial wealth $\bar{x}_{0}>0$, the stochastic differential equation (6) under $\theta^{*}(t, i)$ and $c^{*}(t, x, i)$ has a unique nonnegative solution $\bar{X}^{\theta^{*}, c^{*}}(t)$. Furthermore,

$$
\mathrm{E}\left(\sup _{\mathrm{t} \in[0, \mathrm{~T}]}\left|\overline{\mathrm{X}}^{\theta^{*}, \mathrm{c}^{*}}(\mathrm{t})\right|^{\alpha}\right)<+\infty, \quad \forall \alpha \in \mathbb{R} .
$$

Proof. Substituting (22) and (23) into (6) yields

$$
\begin{aligned}
& d\left(\bar{X}^{\theta^{*}, c^{*}}(t)\right)=\bar{X}^{\theta^{*}, c^{*}}(t)\{\varpi(t, i) d t \\
& +\frac{\kappa(t, i)}{(1-\gamma) \sigma(t, i)} d W(t) \\
& \left.-\sigma_{I}(t, i) \sqrt{1-\rho^{2}(t)} d W_{0}(t)\right\},
\end{aligned}
$$

where

$$
\begin{aligned}
\varpi(t, i)= & \eta(t, i) \\
& +\frac{\kappa(t, i)+(1-\gamma) \rho(t) \sigma(t, i) \sigma_{I}(t, i)}{(1-\gamma) \sigma^{2}(t, i)} \kappa(t, i) \\
& -\left(\frac{\zeta(i)}{\bar{\zeta}(t, i)}\right)^{1 /(1-\gamma)} .
\end{aligned}
$$

Since the coefficients of (41) are uniformly bounded, it is obvious that there exists a unique solution to (41) such as

$$
\begin{aligned}
& \bar{X}^{\theta^{*}, c^{*}}(t)=\bar{x}_{0} \\
& \cdot \exp \left\{\int_{0}^{t}\left[\omega(s, \xi(s))-\frac{1}{2}\left(\frac{\kappa(s, \xi(s))}{(1-\gamma) \sigma(s, \xi(s))}\right)^{2}\right] d s\right.
\end{aligned}
$$

$$
\begin{aligned}
& -\int_{0}^{t} \frac{1}{2}\left(\sigma_{I}(s, \xi(s))\right)^{2}\left(1-\rho^{2}(s)\right) d s \\
& +\int_{0}^{t} \frac{\kappa(s, \xi(s))}{(1-\gamma) \sigma(s, \xi(s))} d W(s) \\
& \left.-\int_{0}^{t} \sigma_{I}(s, \xi(s)) \sqrt{1-\rho^{2}(s)} d W_{0}(s)\right\} .
\end{aligned}
$$

Therefore, $\bar{X}^{\theta^{*}, c^{*}}(t)>0$ for all $t \in[0, T]$.

Next, we shall prove that $\mathrm{E}\left(\sup _{t \in[0, T]}\left|\bar{X}^{\theta^{*}, c^{*}}(t)\right|^{\alpha}\right)<+\infty$ for $\alpha \in \mathbb{R}$. To this end, define $Z(t)=\exp \left\{\int_{0}^{t} h(s, \xi(s))^{\prime} d \widetilde{W}(s)\right\}$, where $\widetilde{W}(t)$ is an $n$-dimensional standard Brownian motion and $h(t, i)$ is an $n \times 1$ column vector whose components are uniformly bounded in $[0, T]$ for any $i \in S$. For $Z(t)$, we have

$$
\begin{aligned}
& Z(t)=\exp \left\{\int_{0}^{t} h(s, \xi(s))^{\prime} d \widetilde{W}(s)\right\} \\
& =\exp \left\{\int_{0}^{t} \frac{1}{2}\|h(s, \xi(s))\|^{2} d s\right\} \\
& \quad \times \exp \left\{-\int_{0}^{t} \frac{1}{2}\|h(s, \xi(s))\|^{2} d s\right. \\
& \left.+\int_{0}^{t} h(s, \xi(s))^{\prime} d \widetilde{W}(s)\right\} \leq H_{1} \\
& \cdot \exp \left\{-\int_{0}^{t} \frac{1}{2}\|h(s, \xi(s))\|^{2} d s\right. \\
& \left.+\int_{0}^{t} h(s, \xi(s))^{\prime} d \widetilde{W}(s)\right\}:=H_{1} \widetilde{Z}(t) .
\end{aligned}
$$

The stochastic differential equation of $\widetilde{Z}(t)$ is of this form:

$$
d \widetilde{Z}(t)=\widetilde{Z}(t) h(t, \xi(t))^{\prime} d \widetilde{W}(t) .
$$

The uniformly bounded $h(t, i)$ results in $\|\widetilde{Z}(t) h(t, \xi(t))\|^{2} \leq$ $H_{2}|\widetilde{Z}(t)|^{2}$; then, according to Krylov [51, p. 85], we have $\mathrm{E}\left(\sup _{t \in[0, T]}|\widetilde{Z}(t)|\right)<+\infty$. It follows $Z(t) \leq H_{1} \widetilde{Z}(t)$ that

$$
\mathrm{E}\left(\sup _{t \in[0, T]} \exp \left(\int_{0}^{t} h(s, \xi(s))^{\prime} d \widetilde{W}(s)\right)\right)<+\infty,
$$

where $h(t, i)$ is any $n \times 1$ column vector whose components are uniformly bounded in $[0, T]$ for any $i \in S$. In view of (43), for any given $\alpha \in \mathbb{R}$ we have

$$
\leq H_{3} \exp \left\{\int_{0}^{t} \frac{\alpha \kappa(s, \xi(s))}{\left(\bar{X}^{\theta^{*}, c^{*}}(t)\right)^{\alpha}} d W(s)-\int_{0}^{t} \alpha \sigma_{I}(s, \xi(s)) \sqrt{1-\rho^{2}(s)} d W_{0}(s)\right\} .
$$

It follows (46) that $\mathrm{E}\left(\sup _{t \in[0, T]}\left(\bar{X}^{\theta^{*}, c^{*}}(t)\right)^{\alpha}\right)<+\infty$.

Lemma 5. $\theta^{*}(t, i)$ in (22) and $c^{*}(t, x, i)$ in (23) are admissible and then are optimal strategies for the power utility model.
Proof. By Lemma 4, we know that conditions (i) and (ii) in Definition 1 hold and $X^{\theta^{*}, c^{*}}(t)>0$ for all $t \in[0, T]$, which guarantees (iv) in Definition 1 holds. Since $\theta^{*}(t, i)$ and $\zeta(i) / \bar{\zeta}(t, i)$ are time deterministic and uniformly bounded 
functions for any given market state $i, \mathrm{E}\left(\int_{0}^{T}\left|\theta^{*}(t, \xi(t))\right|^{2}\right)<$ $+\infty$ holds naturally. By Lemma 4 , we have

$$
\begin{aligned}
& \mathrm{E}\left(\int_{0}^{T}\left|c^{*}\left(t, \bar{X}^{\theta^{*}, c^{*}}(t), \xi(t)\right)\right|^{\gamma} d t\right) \\
& \quad=\mathrm{E}\left(\int_{0}^{T}\left(\bar{X}^{\theta^{*}, c^{*}}(t)\right)^{\gamma}\left(\frac{\zeta(\xi(t))}{\bar{\zeta}(t, \xi(t))}\right)^{\gamma /(1-\gamma)} d t\right) \\
& \quad \leq M_{1} \mathrm{E}\left(\int_{0}^{T}\left(\bar{X}^{\theta^{*}, c^{*}}(t)\right)^{\gamma} d t\right) \\
& \quad \leq M_{1} \mathrm{E}\left(\int_{0}^{T} \sup _{t \in[0, T]}\left(\bar{X}^{\theta^{*}, c^{*}}(t)\right)^{\gamma} d t\right)<+\infty .
\end{aligned}
$$

Now we have verified that $c^{*}(t, x, i)$ and $\theta^{*}(t, i)$ are admissible and hence optimal for the power utility model.

The next work is to prove that the candidate value function $v(t, x, i)$ in $(20)$ satisfies all the conditions in Theorem 2. First of all, it is obvious that $v(t, x, i) \in C^{1,2}$ is a solution of (9). Moreover, for any $(t, x, i) \in[0, T] \times[0,+\infty) \times S$ and admissible control $(\theta(t), c(t))$, there exists a $\beta=2>1$ such that

$$
\begin{aligned}
& \mathrm{E}\left(\sup _{s \in[t, T]}\left|v\left(s, \bar{X}^{\theta, c}(s), \xi(s)\right)\right|^{\beta}\right) \\
& =\mathrm{E}\left(\sup _{s \in[t, T]}\left|\bar{\zeta}(s, \xi(s)) \frac{\left(\bar{X}^{\theta, c}(s)\right)^{\gamma}}{\gamma}\right|^{2}\right) \\
& \quad \leq M_{2} \mathrm{E}\left(\sup _{s \in[t, T]}\left(\bar{X}^{\theta, c}(s)\right)^{2 \gamma}\right)<+\infty .
\end{aligned}
$$

The detailed analysis above gives the main results of this paper presented in the following theorem.

Theorem 6. The optimal investment proportion and the optimal consumption for the power utility model are, respectively,

$$
\begin{aligned}
\theta^{*}(t, i) & =\frac{\tilde{\mu}(t, i)}{(1-\gamma) \sigma^{2}(t, i)}-\frac{\gamma}{1-\gamma} \frac{\rho(t) \sigma_{I}(t, i)}{\sigma(t, i)}, \\
c^{*}(t, x, i) & =\left(\frac{\zeta(i)}{\bar{\zeta}(t, i)}\right)^{1 /(1-\gamma)} x,
\end{aligned}
$$

where $\bar{\zeta}(t, i)$ solves (24) and the value function is

$$
V(t, x, i)=\frac{\bar{\zeta}(t, i) x^{\gamma}}{\gamma}
$$

\section{Analysis of the Optimal Investment Proportion}

First of all, if there is no inflation, by (50), the optimal investment proportion is

$$
\theta^{*}(t, i)=\frac{\tilde{\mu}(t, i)}{(1-\gamma) \sigma^{2}(t, i)},
$$

which clearly shows that when the market state has higher expected return per unit risk or the investor has lower risk aversion, the investor would like to invest higher proportion of his wealth on the stock, which is a classical conclusion in the existing literature if the investor does not need to face the inflation.

However, when there is inflation, this conclusion may not hold. First, we can prove that the higher expected return per unit risk does not result in a higher investment proportion. By (50), the investment proportion is decreased by an amount of $(\gamma /(1-\gamma)) \rho(t) \sigma_{I}(t, i) / \sigma(t, i)$ compared with the portfolio selection without inflation. This amount is increased with respect to the volatility rate of the inflation and the correlation coefficient $\rho(t)$. When $\rho(t) \equiv 1$, that is, the stock price and the inflation index are modulated by the same Brownian motion, the investment proportion is decreased by the largest amount. That means, if the stock and the commodity price level have the same volatility trend, the inflation volatility will diminish the investment proportion the most. Therefore, when the increasing range of the expected return per unit is lower than that of the inflation volatility, the investor would not buy more stocks and could even short sell the stock because he worries the high volatility of the inflation would seriously damage his investment return.

Next, we shall present the effects of the risk aversion on the investment proportion.

Lemma 7. When $\widetilde{\mu}(t, i)>\rho(t) \sigma_{I}(t, i) \sigma(t, i)$, the optimal investment proportion is increased with respect to the risk tolerance $1 /(1-\gamma)$; when $\widetilde{\mu}(t, i)<\rho(t) \sigma_{I}(t, i) \sigma(t, i)$, the optimal investment proportion is decreased with respect to the risk tolerance; when $\widetilde{\mu}(t, i)=\rho(t) \sigma_{I}(t, i) \sigma(t, i)$, the optimal investment proportion is a constant $\rho(t) \sigma_{I}(t, i) / \sigma(t, i)$.

Proof. We rewrite (50) as

$$
\begin{aligned}
\theta^{*}(t, i)= & \frac{1}{1-\gamma} \frac{\widetilde{\mu}(t, i)-\rho(t) \sigma_{I}(t, i) \sigma(t, i)}{\sigma^{2}(t, i)} \\
& +\frac{\rho(t) \sigma_{I}(t, i)}{\sigma(t, i)} ;
\end{aligned}
$$

it is clear that the conclusions of Lemma 7 hold.

Remark 8. When $\sigma_{I}(t, i)=0, \widetilde{\mu}(t, i)>0$ holds naturally. Therefore, the investment proportion increases as the risk tolerance increases, which reduces to a classical conclusion in the model without inflation.

Remark 9. When there is no inflation, the investment proportion $\theta^{*}(t, i)$ is a positive number if $\widetilde{\mu}(t, i)>0$. However, this conclusion does not hold in the case with inflation even if $\widetilde{\mu}(t, i)>\rho(t) \sigma_{I}(t, i) \sigma(t, i)$. When $0<\gamma<1$, that is, the risk tolerance is greater than $1, \widetilde{\mu}(t, i)>\rho(t) \sigma_{I}(t, i) \sigma(t, i)$ leads to $\tilde{\mu}(t, i)>\gamma \rho(t) \sigma_{I}(t, i) \sigma(t, i)$. By $(50), \theta^{*}(t, i)>0$. When $\gamma<0$, that is, the risk tolerance is less than $1, \widetilde{\mu}(t, i)>$ $\rho(t) \sigma_{I}(t, i) \sigma(t, i)$ cannot always guarantee a positive investment proportion if $\rho(t)<0$. 
Remark 10. If $0<\tilde{\mu}(t, i)<\rho(t) \sigma(t, i) \sigma_{I}(t, i)$, the investment proportion will decrease according to the risk tolerance. Moreover, if the risk tolerance is high enough, the investor will tend to short sell her/his stock, and the short selling proportion is increasing according to the risk tolerance.

\section{Analysis of the Optimal Consumption Proportion}

Denote by

$$
\operatorname{cp}(t, i):=\left(\frac{\zeta(i)}{\bar{\zeta}(t, i)}\right)^{1 /(1-\gamma)}
$$

the consumption proportion. Next, we shall analyze in detail the effects of the risk aversion, the correlation coefficient, the expected rate and volatility rate of the inflation index, and the utility coefficients on the consumption proportion. We assume that the risk-free return rate is a constant $r=0.02$ independent of time and market states, and the appreciation rate $\mu$ and volatility rate $\sigma$ of the stock depend on the market states only. Let there be two market states, and $\mu(1)=0.2$, $\mu(2)=0.15, \sigma(1)=0.25, \sigma(2)=0.4$, the discount rate $\delta=0.8$, the time horizon $T=5$, and the generator

$$
q=\left(\begin{array}{cc}
-2.5 & 2.5 \\
4 & -4
\end{array}\right)
$$

5.1. Effects of the Risk Aversion. In this subsection, assume that $\rho=0.4, \mu_{I}=(0.05,0.05), \sigma_{I}=(0.15,0.15)$, and $\zeta=$ $(1,1)$. We increase $\gamma$ from -0.4 to 0.95 with step size 0.1 . Then, the effects of risk aversion on the consumption proportion are obtained as demonstrated in Figure 1.

Figure 1 shows the following:

(i) As $t \rightarrow T$, consumption proportion approaches 1 , which is consistent with the conclusion in Cheung and Yang [30].

(ii) As $\gamma$ is increased from -0.4 to some extent, the consumption proportion is raised accordingly. However, there come changes when $\gamma$ continues to increase. The consumption proportions almost decrease to 0 as $\gamma$ increases to 0.95 . Actually, since now $\kappa(t, \cdot)=$ $\tilde{\mu}(t, \cdot)-\rho(t) \sigma_{I}(t, \cdot) \sigma(t, \cdot)=(0.165,0.106)$, according to Lemma 7, an investor with higher risk tolerance $1 /(1-$ $\gamma$ ) will invest more of her/his wealth in the stock and consequently consume less of her/his wealth. That is, when $\gamma$ is close to 1 , the consumption proportion is almost zero in most cases.

(iii) When $\gamma$ is relatively small, the investor consumes a larger proportion of our wealth if it is closer to the end of the horizon. When $\gamma$ is close to 1 , that is, the risk tolerance is relatively high, the consumption rate decreases with time.

\subsection{Effects of the Correlation Coefficients}

Lemma 11. When $\rho(t)$ is a constant $\rho$ in $[0, T]$ and $\gamma<0$, the consumption proportion $c p(t, i)$ is increasing according to the correlation coefficient $\rho$ if $\tilde{\mu}(s, j)>\gamma \rho \sigma(s, j) \sigma_{I}(s, j)$ for all $j \in S$ and $s \in[t, T]$.

Proof. By (25), we have

$$
\frac{\partial \phi}{\partial \rho}=-\frac{\gamma^{2}}{1-\gamma} \frac{\sigma_{I}(t, i)}{\sigma(t, i)}\left[\widetilde{\mu}(t, i)-\gamma \rho \sigma(t, i) \sigma_{I}(t, i)\right] .
$$

If $\tilde{\mu}(s, j)>\gamma \rho \sigma(s, j) \sigma_{I}(s, j)$ for all $j \in S$ and $s \in[t, T]$, we know that $\phi(s, j)$ decreases with respect to $\rho$ in $s \in$ $[t, T]$, which has a consequence that $K(t, s)$ in (26) decreases accordingly if $\rho$ increases for all $s \in[t, T]$. When $\gamma<0$, $0<\gamma /(\gamma-1)<1$. Therefore, $(\bar{\zeta}(t, i))^{\gamma /(\gamma-1)}$ is an increasing function of $\bar{\zeta}(t, i)$. This together with the Picard sequence (31) indicates that $\left\{\bar{\zeta}^{(k)}: k=0,1,2, \ldots\right\}$ decreases as $K(t, i)$ decreases. Since $\bar{\zeta}(t, i)$ is the limit of the Picard sequence, we immediately obtain that $\bar{\zeta}(t, i)$ decreases as $\rho$ increases. Now it follows (55) that the conclusion in Lemma 11 holds.

Let $\gamma=-0.8$ and increase the correlation coefficient $\rho$ from -1 to 1 with step size 0.5 while keeping other parameters unchangeable. Since the minimal value of $\widetilde{\mu}(t, \cdot)-$ $\gamma \rho \sigma(t, \cdot) \sigma_{I}(t, \cdot)$ is $(0.150,0.082)$, we can see clearly in Figure 2 that the consumption proportion at state 1 increases according to the increasing correlation coefficients. However, if we assume that $r=0.14, \mu=(0.16,0.15)$, and $\gamma=-4$, then $\tilde{\mu}(t, j)<\rho \gamma \sigma(t, j) \sigma_{I}(t, j)$ given that $\rho=-1$ and -0.5 . Therefore, we obtain Figure 3, which shows that the higher the $\rho$ is, the lower the consumption proportion cp is.

\subsection{Effects of the Expected Inflation Rate}

Lemma 12. The consumption proportion $c p(t, i)$ decreases if the expected inflation rate $\mu_{I}(s, j)$ increases for all $j \in S$ and $s \in[t, T]$ when $\gamma<0$.

Proof. The proof of Lemma 12 is similar to that of Lemma 11, so it is omitted here.

Let $\gamma=-0.5$ and $\rho=0.4$ and increase respectively $\mu_{I}(1)$ and $\mu_{I}(2)$ from 0.05 to 0.15 with step size 0.02 while keeping other parameters unchangeable; we obtain Figure 4. But if we change $\gamma$ to be 0.5 while keeping other parameters unchangeable, we obtain Figures 5 and 6.

Figures $4-6$ show that if the risk aversion $1-\gamma$ is greater than 1 , then the higher the expected inflation rate is, the lower the consumption proportion is; otherwise, if the risk aversion $1-\gamma$ is less than 1 , the best decision for the investor is to consume a high proportion of her/his wealth at the current time when the expected inflation rate in the future is high no matter what the market state is.

5.4. Effects of the Inflation Volatility. Let $\gamma=0.8, \rho=0.4$, $\sigma_{I}(2)=0.15, \mu_{I}=(0.05,0.05)$, and $\zeta=(1,1)$ and increase $\sigma_{I}(1)$ from 0.15 to 0.25 with step size 0.02 . The effects of the volatility of inflation on the consumption proportion are demonstrated in Figure 7. One can see that the higher the volatility rate is, the more the investor consumes. A similar 

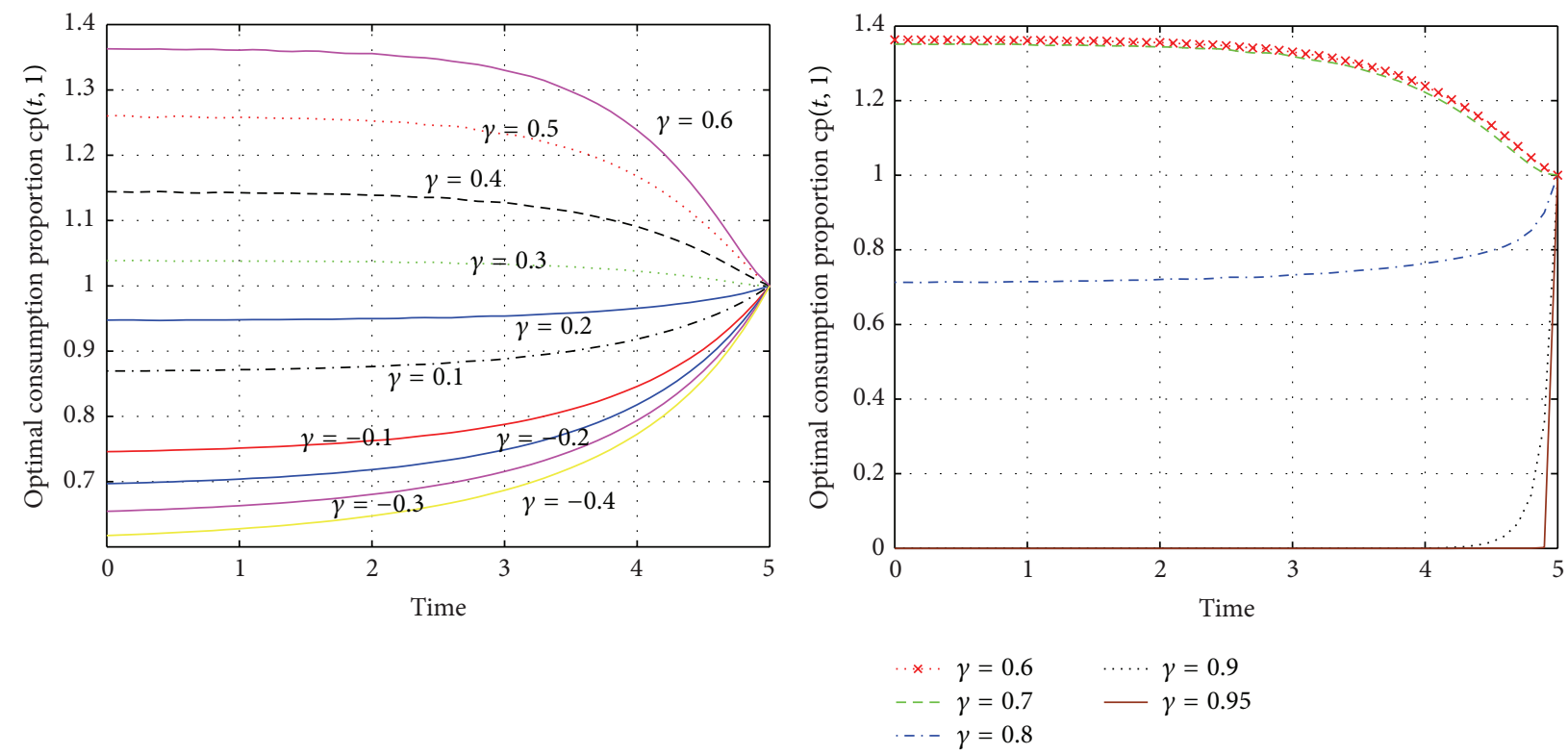

(a)

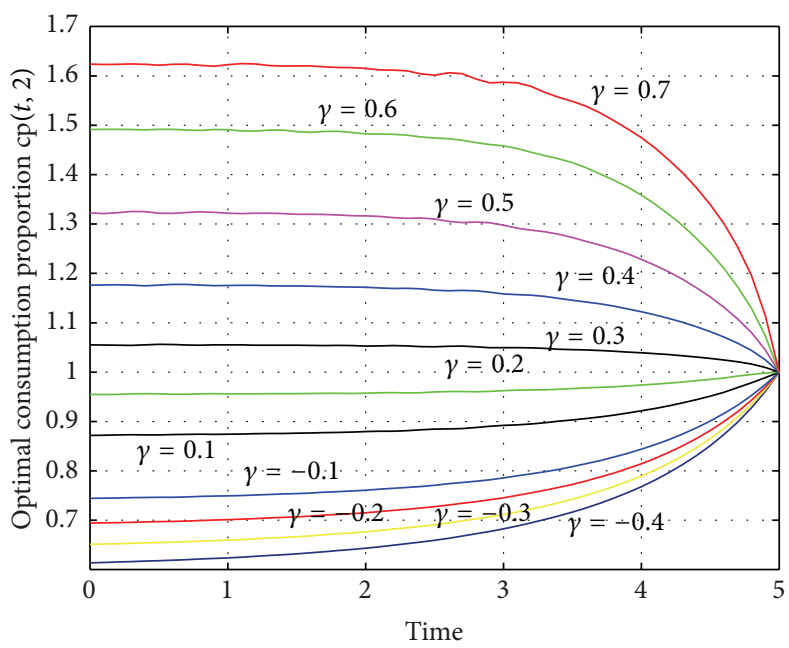

(c)

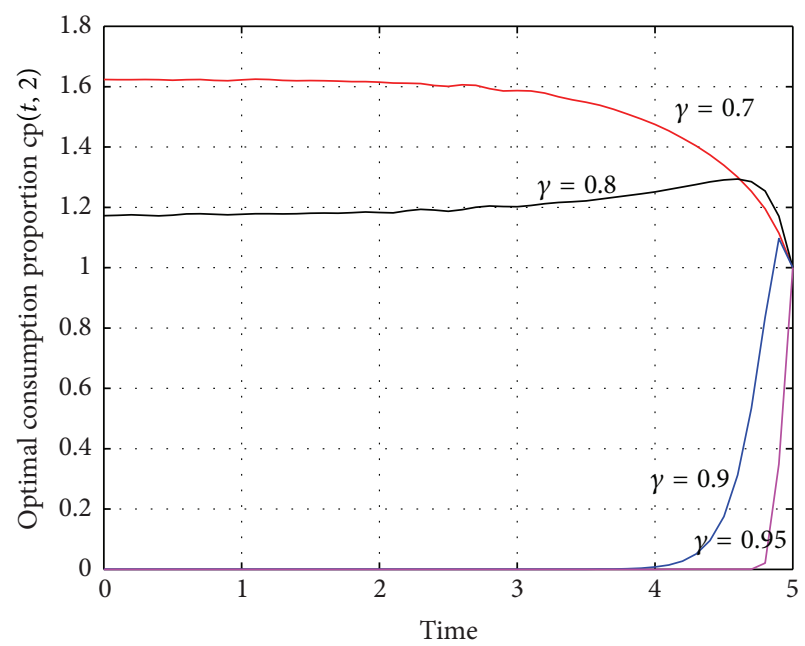

(d)

FIGURE 1: Consumption proportion with respect to $\gamma$.

phenomenon happens when we increase $\sigma_{I}(2)$ from 0.15 to 0.25 with step size 0.02 while keeping $\sigma_{I}(1)=0.15$. To explain this, we notice that $\gamma>0$ in Figure 7, which has a consequence that the higher the volatility rate $\sigma_{I}(t, i)$ is, the lower the investment proportion is, by (50). Therefore, more wealth is used for personal consumption.

5.5. Effects of the Utility Coefficient. In this subsection, let $\mu_{I}=(0.05,0.05), \sigma_{I}=(0.15,0.15), \gamma=0.6$, and $\rho=0.4$ and increase $\zeta(1)$ and $\zeta(2)$ from 0.2 to 1 with step size 0.2 , respectively. Then, we have Figures 8 and 9 .

Figures 8 and 9 present an interesting phenomenon that the increasing $\zeta(i)$ results in an increasing $\mathrm{cp}(t, i)$ and a decreasing $\operatorname{cp}(t, j), j \neq i$. Actually, we can regard $\zeta(i)$ as the attention degree of the consumption at state $i$. Hence, a larger $\zeta(i)$ indicates that the investor cares more about the consumption utility at state $i$ and hence consumes a larger amount of her/his wealth. In contrast, the consumption proportion at other market states will be diminished correspondingly.

\section{Conclusion}

This paper considers a continuous-time investment-consumption problem under inflation where the stock price, the commodity price level, and the coefficient of the power utility all depend on the market states. The admissible strategy 


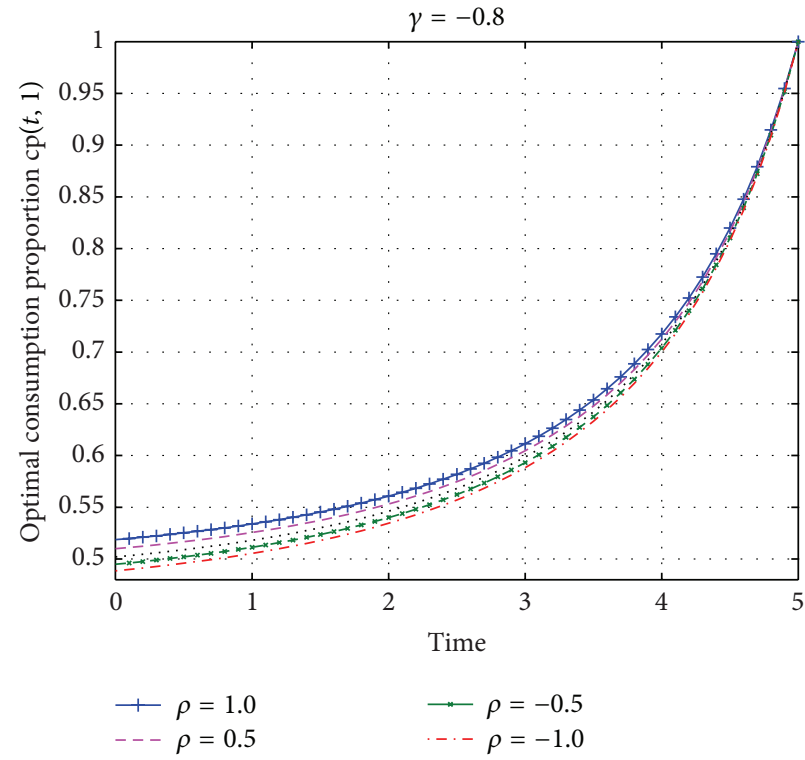

(a)

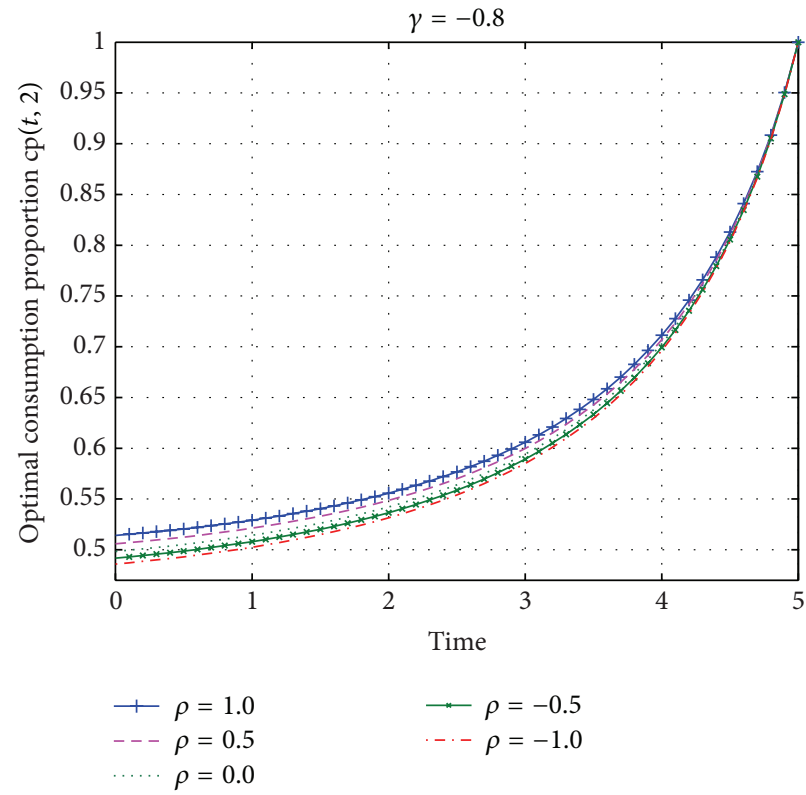

(b)

FIGURE 2: Consumption proportion with respect to $\rho$.

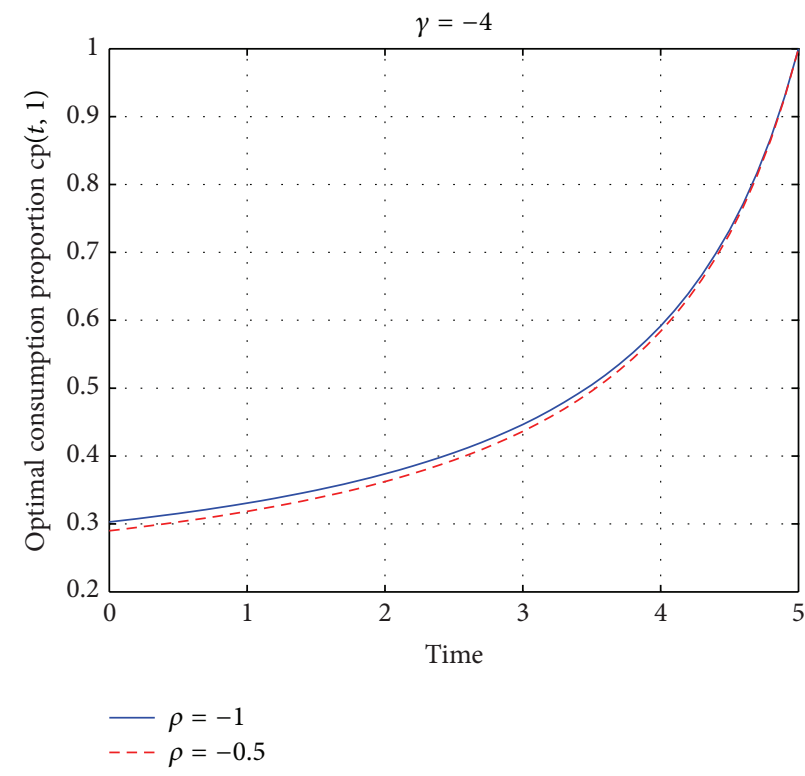

(a)

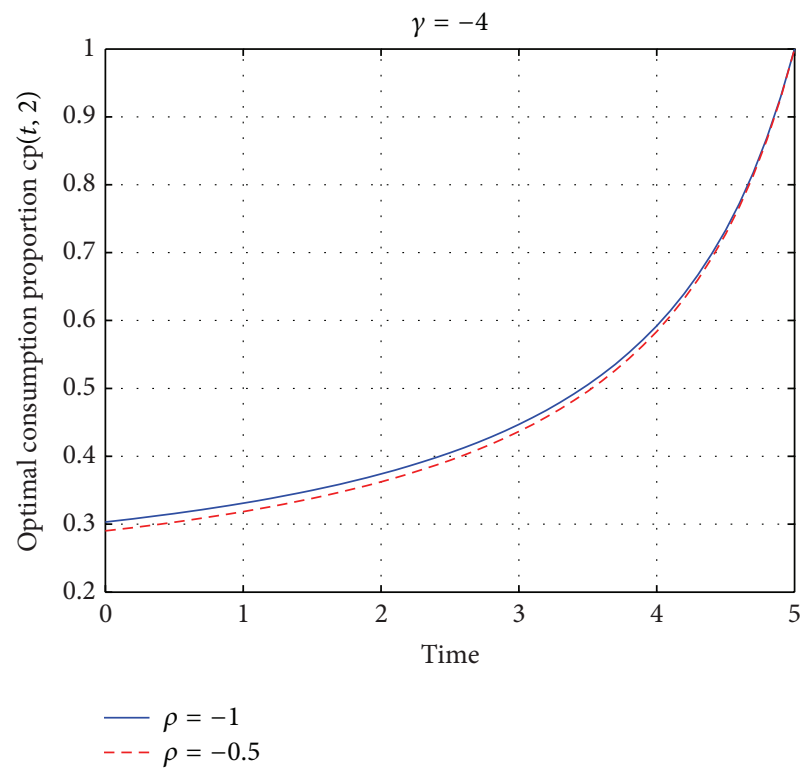

(b)

FIgURE 3: Consumption proportion with respect to $\rho$.

and verification theory corresponding to this problem are provided. We obtain the closed-form investment strategy and quasiexplicit consumption strategy by dynamic programming and stochastic control technique. By mathematical and numerical analysis, we obtain some interesting properties of the optimal strategies.
For the optimal strategy, (a) we say that a market has a better state if at this state the stock has a higher expected excess return per unit risk (the Sharpe ratio). Under the influence of the inflation, the investor would not always invest more wealth in the stock even if the market state is better. If the increasing range of the inflation volatility is higher than 


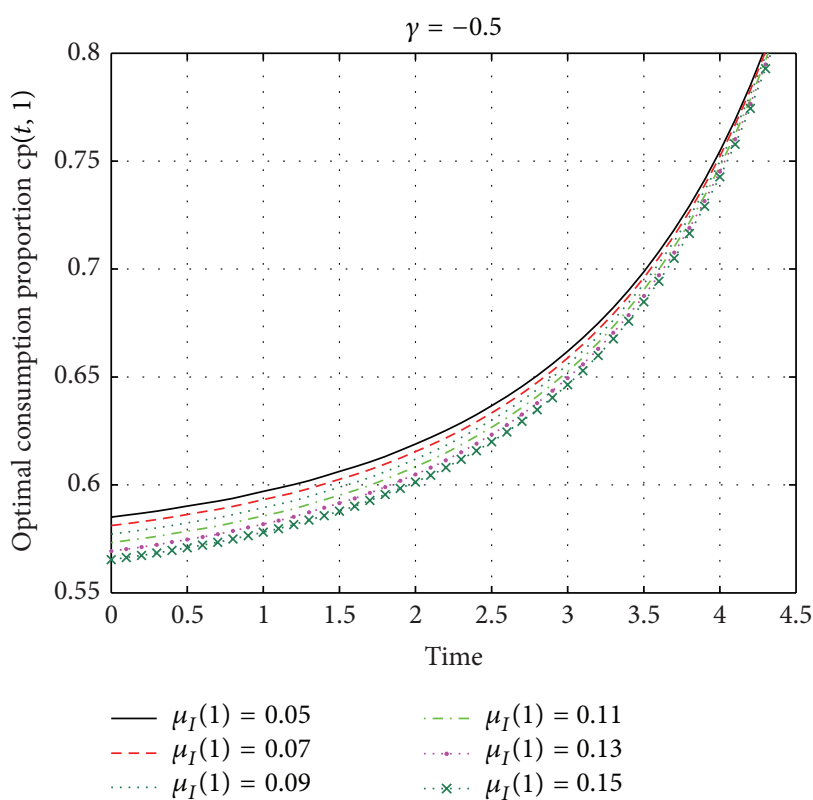

(a)

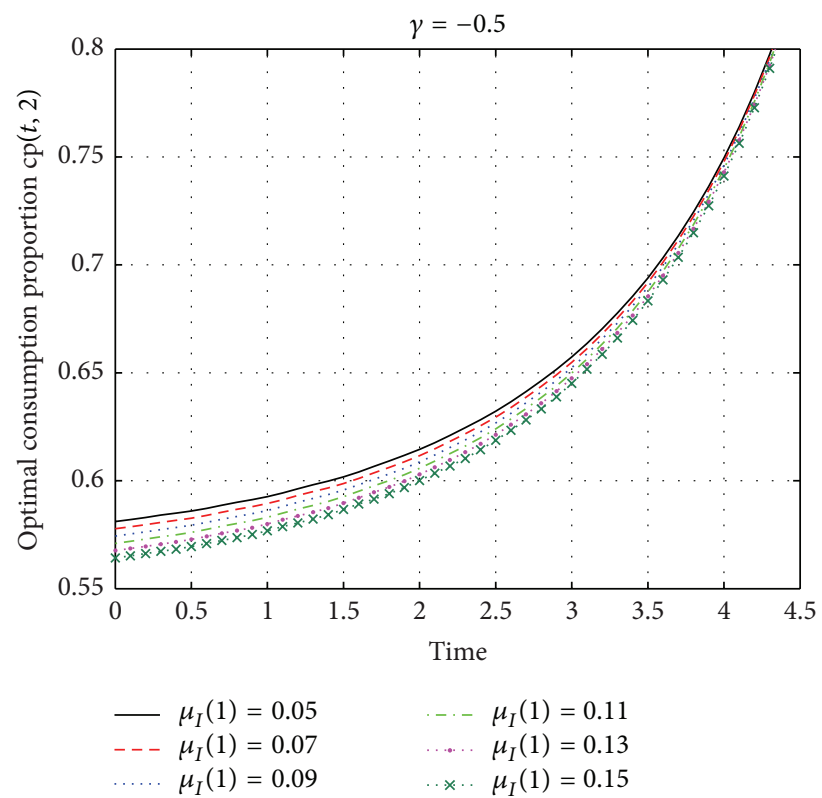

(b)

FIgURE 4: Consumption proportion with respect to $\mu_{I}(1)$.

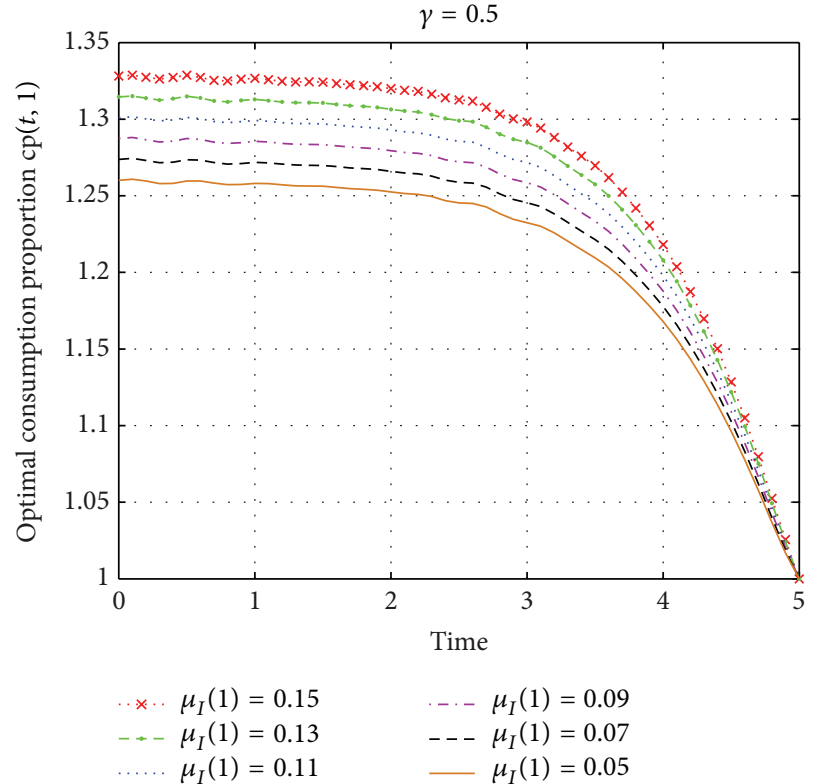

(a)

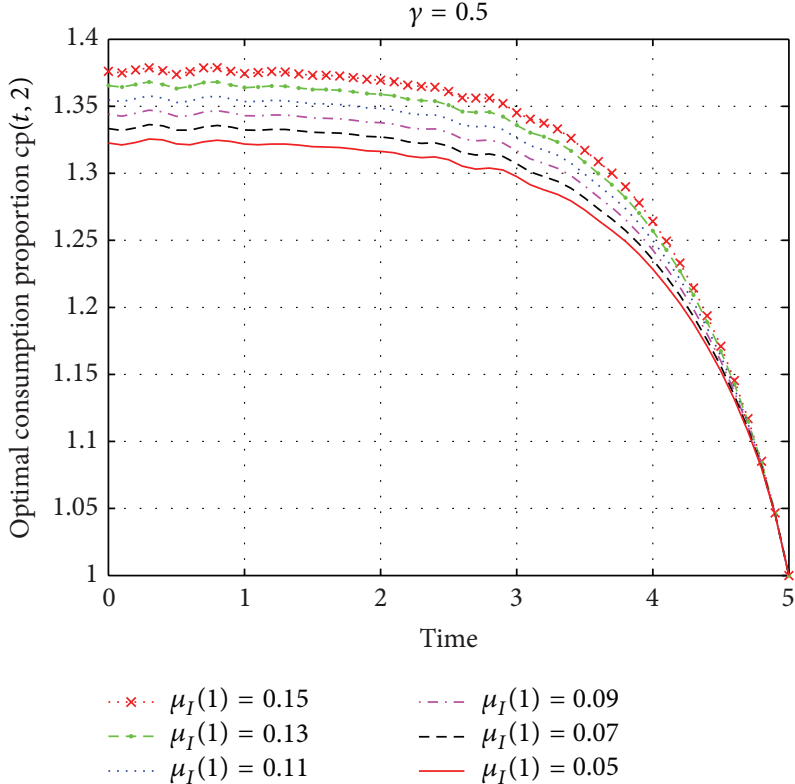

(b)

FIGURE 5: Consumption proportion with respect to $\mu_{I}(1)$.

that of the Sharpe ratio of the stock, the investor would not invest more of his wealth on this stock since the high inflation erodes greatly the investment enthusiasm of the investor even if he is at a better market state; (b) if there is no inflation, then when the Sharpe ratio is greater than 0 , an investor with higher risk aversion would invest less of his wealth in the stock. But if there exists inflation, the positive Sharpe ratio cannot guarantee this conclusion holding. Only if the Sharpe ratio is greater than the product of inflation volatility rate and correlation coefficient $\rho(t)$ does the traditional conclusion hold; (c) the expected inflation rate and the utility coefficient have no impact on the optimal investment strategy.

For the optimal consumption strategy, (a) when the risk aversion is close to zero, the consumption proportion is almost zero. When the risk aversion is relatively small (big), the consumption proportion decreases (increases) with time; 


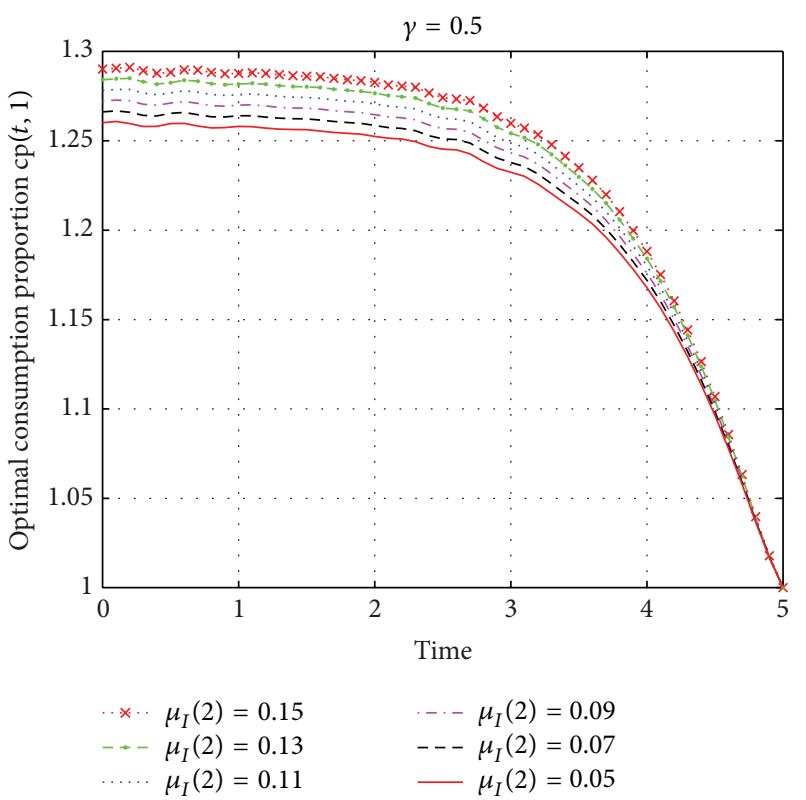

(a)

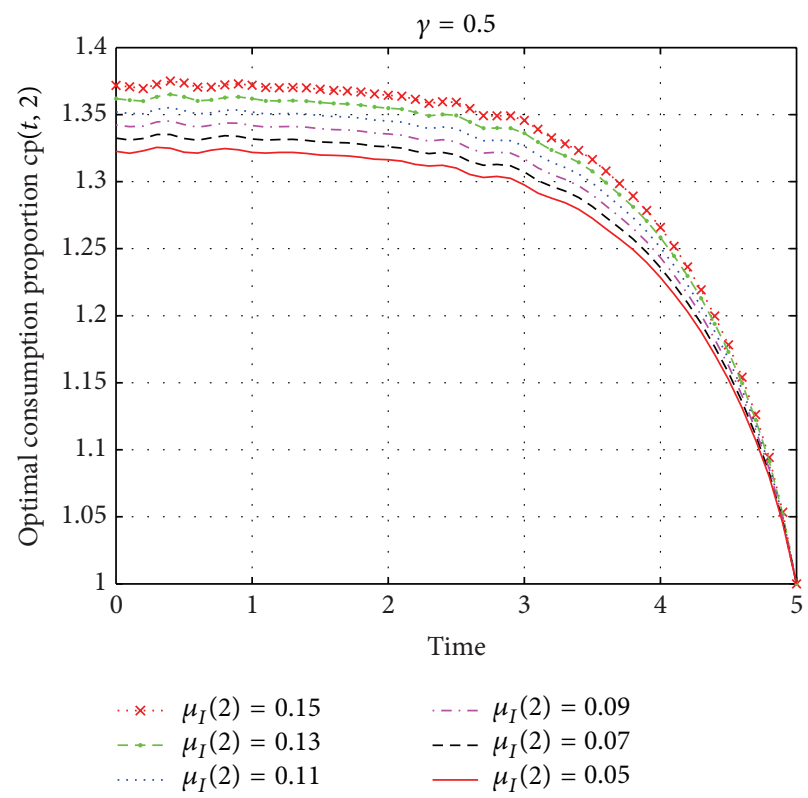

(b)

FIGURE 6: Consumption proportion with respect to $\mu_{I}(2)$.

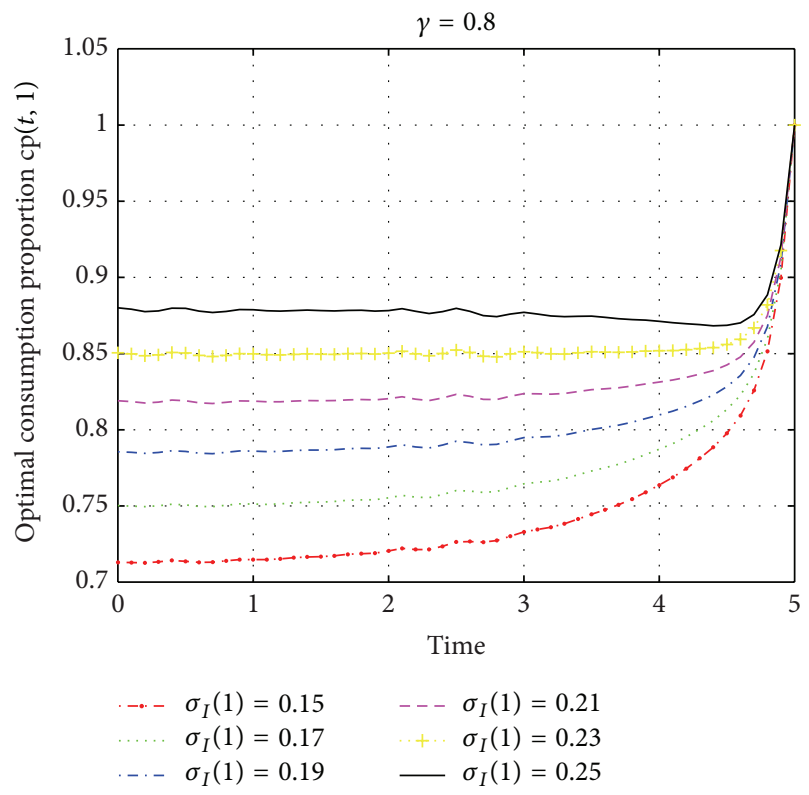

(a)

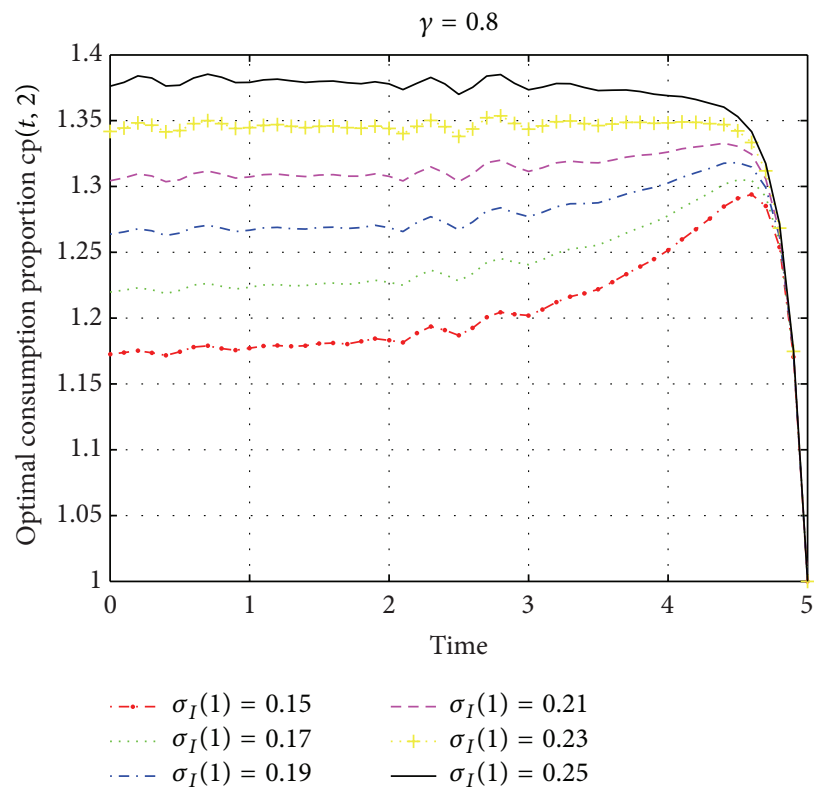

(b)

FIgURE 7: Consumption proportion with respect to $\sigma_{I}(1)$.

(b) when correlation coefficient $\rho(t)$ is a constant in $[0, T]$ and the risk aversion is greater than 1 , the consumption proportion is increasing according to the correlation coefficient if the Sharpe ratio of the stock is high enough; (c) when the risk aversion is greater than 1 , the consumption proportion decreases according to an increasing expected inflation rate; (d) the higher the volatility rate of the inflation is, the higher the consumption proportion is; (e) a larger coefficient of utility $\zeta(i)$ results in a higher consumption proportion at state $i$ but a lower consumption proportion at state $j \neq i$.

Although our model is rather general, it still deserves further extension as future research. For example, in most existing literature including our paper, only the coefficient of the utility depends on the market states, but the risk aversion 


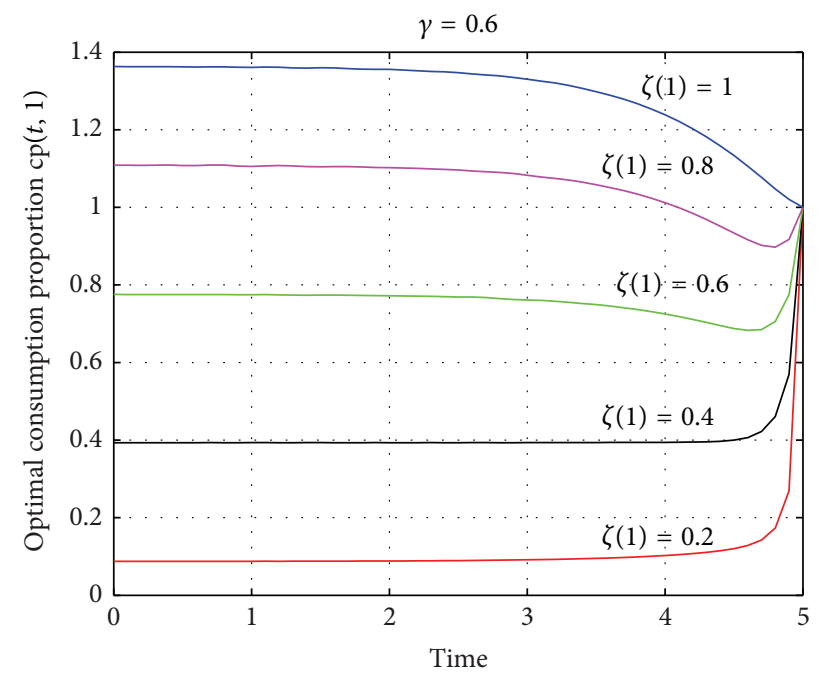

(a)

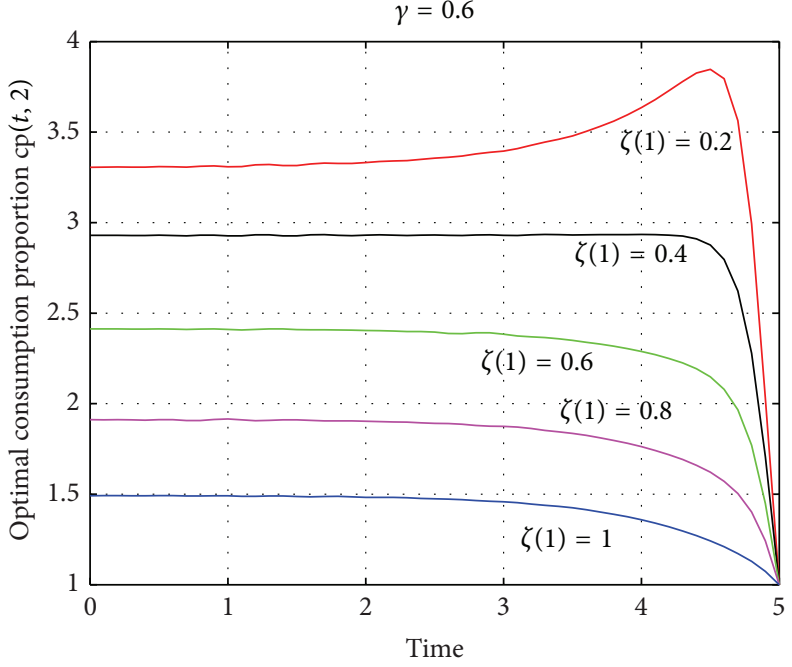

(b)

Figure 8: Consumption proportion with respect to $\zeta(1)$.

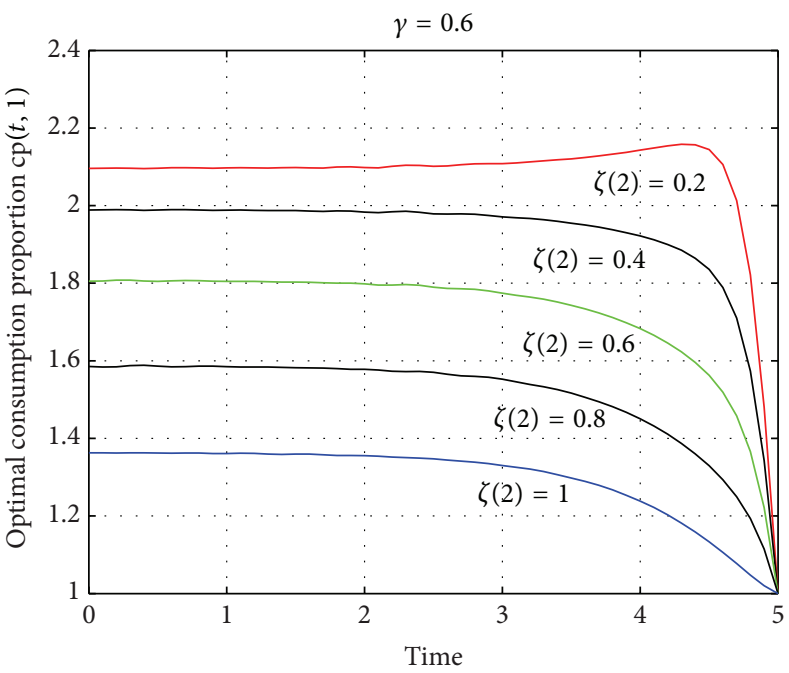

(a)

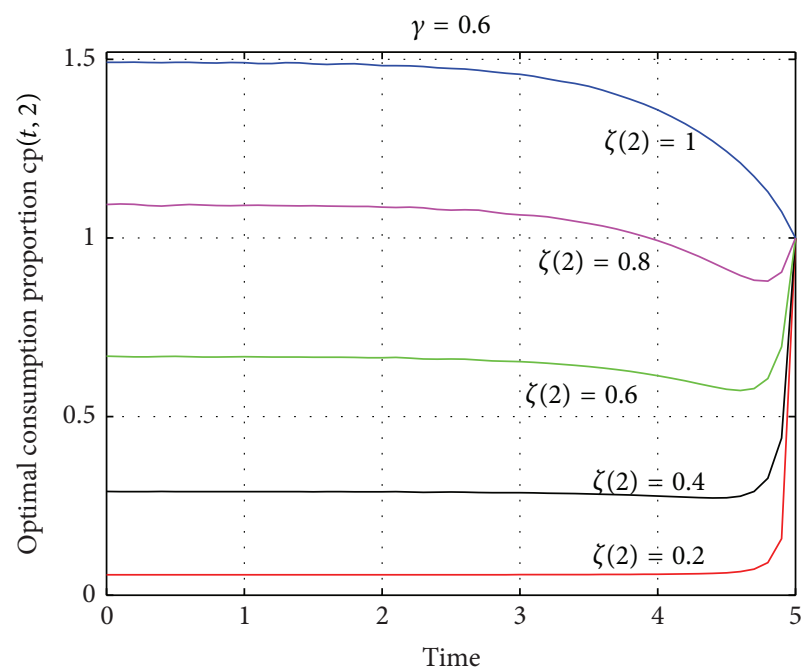

(b)

FIGURE 9: Consumption proportion with respect to $\zeta(2)$.

is independent of the market state. So the future research may focus on the optimal investment-consumption problem with a state-dependent risk aversion.

\section{Competing Interests}

The author declares that they have no competing interests.

\section{Acknowledgments}

This research is supported by grants of the National Natural Science Foundation of China (no. 11301562), the Program for Innovation Research in Central University of Finance and Economics, and Beijing Social Science Foundation (no. 15JGB049).

\section{References}

[1] P. A. Samuelson, "Lifetime portfolio selection by dynamic stochastic programming," The Review of Economics and Statistics, vol. 51, no. 3, pp. 239-246, 1969.

[2] N. H. Hakansson, "Optimal investment and consumption strategies under risk for a class of utility functions," Econometrica, vol. 38, no. 5, pp. 587-607, 1970.

[3] E. F. Fama, "Multiperiod consumption-investment decisions," The American Economic Review, vol. 60, no. 1, pp. 163-174, 1970.

[4] R. C. Merton, "Optimum consumption and portfolio rules in a continuous-time model," Journal of Economic Theory, vol. 3, no. 4, pp. 373-413, 1971.

[5] T. Zariphopoulou, "Investment-consumption models with transaction fees and Markov-chain parameters," SIAM Journal on Control and Optimization, vol. 30, no. 3, pp. 613-636, 1992. 
[6] M. Akian, J. L. Menaldi, and A. Sulem, "On an investmentconsumption model with transaction costs," SIAM Journal on Control and Optimization, vol. 34, no. 1, pp. 329-364, 1996.

[7] H. Liu, "Optimal consumption and investment with transaction costs and multiple risky assets," The Journal of Finance, vol. 59, no. 1, pp. 289-338, 2004.

[8] X.-Y. Zhao and Z.-K. Nie, "Multi-asset investment-consumption model with transaction costs," Journal of Mathematical Analysis and Applications, vol. 309, no. 1, pp. 198-210, 2005.

[9] M. Dai, L. Jiang, P. Li, and F. Yi, "Finite horizon optimal investment and consumption with transaction costs," SIAM Journal on Control and Optimization, vol. 48, no. 2, pp. 11341154, 2009.

[10] M. Taksar and S. Sethi, "Infinite-horizon investment consumption model with a nonterminal bankruptcy," Journal of Optimization Theory and Applications, vol. 74, no. 2, pp. 333-346, 1992.

[11] T. Zariphopoulou, "Consumption-investment models with constraints," SIAM Journal on Control and Optimization, vol. 32, no. 1, pp. 59-85, 1994.

[12] C. Munk and C. Sørensen, "Optimal consumption and investment strategies with stochastic interest rates," Journal of Banking \& Finance, vol. 28, no. 8, pp. 1987-2013, 2004.

[13] X. K. Wang and Y. Q. Yi, "An optimal investment and consumption model with stochastic returns," Applied Stochastic Models in Business and Industry, vol. 25, no. 1, pp. 45-55, 2009.

[14] C. Munk, "Optimal consumption/investment policies with undiversifiable income risk and liquidity constraints," Journal of Economic Dynamics and Control, vol. 24, no. 9, pp. 1315-1343, 2000.

[15] P. H. Dybvig and H. Liu, "Lifetime consumption and investment: retirement and constrained borrowing," Journal of Economic Theory, vol. 145, no. 3, pp. 885-907, 2010.

[16] S. R. Pliska and J. Ye, "Optimal life insurance purchase and consumption/investment under uncertain lifetime," Journal of Banking \& Finance, vol. 31, no. 5, pp. 1307-1319, 2007.

[17] M. Kwak, Y. H. Shin, and U. J. Choi, "Optimal investment and consumption decision of a family with life insurance," Insurance: Mathematics \& Economics, vol. 48, no. 2, pp. 176-188, 2011.

[18] M. R. Hardy, "A regime-switching model of long-term stock returns," North American Actuarial Journal, vol. 5, no. 2, pp. 4153, 2001.

[19] X. Y. Zhou and G. Yin, "Markowitz's mean-variance portfolio selection with regime switching: a continuous-time model," SIAM Journal on Control and Optimization, vol. 42, no. 4, pp. 1466-1482, 2003.

[20] U. Çakmak and S. Özekici, "Portfolio optimization in stochastic markets," Mathematical Methods of Operations Research, vol. 63, no. 1, pp. 151-168, 2006.

[21] U. Çelikyurt and S. Özekici, "Multiperiod portfolio optimization models in stochastic markets using the mean-variance approach," European Journal of Operational Research, vol. 179, no. 1, pp. 186-202, 2007.

[22] S.-Z. Wei and Z.-X. Ye, "Multi-period optimization portfolio with bankruptcy control in stochastic market," Applied Mathematics and Computation, vol. 186, no. 1, pp. 414-425, 2007.

[23] H. L. Wu and Z. F. Li, "Multi-period mean-variance portfolio selection with Markov regime switching and uncertain timehorizon," Journal of Systems Science and Complexity, vol. 24, no. 1, pp. 140-155, 2011.
[24] H. L. Wu and Z. F. Li, "Multi-period mean-variance portfolio selection with regime switching and a stochastic cash flow," Insurance: Mathematics and Economics, vol. 50, no. 3, pp. 371384, 2012.

[25] H. Wu and Y. Zeng, "Multi-period mean-variance portfolio selection in a regime-switching market with a bankruptcy state," Optimal Control Applications \& Methods, vol. 34, no. 4, pp. 415432, 2013.

[26] P. Chen, H. L. Yang, and G. Yin, "Markowitz's mean-variance asset-liability management with regime switching: a continuous-time model," Insurance: Mathematics and Economics, vol. 43, no. 3, pp. 456-465, 2008.

[27] K. C. Cheung and H. L. Yang, "Asset allocation with regimeswitching: discrete-time case," ASTIN Bulletin, vol. 34, pp. 247257, 2004.

[28] E. Çanakoğlu and S. Ozekici, "Portfolio selection in stochastic markets with HARA utility functions," European Journal of Operational Research, vol. 201, no. 2, pp. 520-536, 2010.

[29] E. Çanakoğlu and S. Ozekici, "HARA frontiers of optimal portfolios in stochastic markets," European Journal of Operational Research, vol. 221, no. 1, pp. 129-137, 2012.

[30] K. C. Cheung and H. Yang, "Optimal investment-consumption strategy in a discrete-time model with regime switching," Discrete and Continuous Dynamical Systems. Series B, vol. 8, no. 2, pp. 315-332, 2007.

[31] Z. Li, K. S. Tan, and H. Yang, "Multiperiod optimal investmentconsumption strategies with mortality risk and environment uncertainty," North American Actuarial Journal, vol. 12, no. 1, pp. 47-64, 2008.

[32] Y. Zeng, H. Wu, and Y. Lai, "Optimal investment and consumption strategies with state-dependent utility functions and uncertain time-horizon," Economic Modelling, vol. 33, pp. 462470, 2013.

[33] P. Gassiat, F. Gozzi, and H. Pham, "Investment/consumption problems in illiquid markets with regime-switching," SIAM Journal on Control and Optimization, vol. 52, no. 3, pp. 17611786, 2014.

[34] T. A. Pirvu and H. Y. Zhang, "Investment and consumption with regime-switching discount rates," Working Paper, http://arxiv .org/abs/1303.1248.

[35] M. J. Brennan and Y. Xia, "Dynamic asset allocation under inflation," The Journal of Finance, vol. 57, no. 3, pp. 1201-1238, 2002.

[36] C. Munk, C. Sørensen, and T. Nygaard Vinther, "Dynamic asset allocation under mean-reverting returns, stochastic interest rates, and inflation uncertainty: are popular recommendations consistent with rational behavior?" International Review of Economics and Finance, vol. 13, no. 2, pp. 141-166, 2004.

[37] C. Chiarella, C. Y. Hsiao, and W. Semmler, Intertemporal Investment Strategies under Inflation Risk, vol. 192 of Research Paper Series, Quantitative Finance Research Centre, University of Technology, Sydney, Australia, 2007.

[38] F. Menoncin, "Optimal real investment with stochastic income: a quasi-explicit solution for HARA investors," Working Paper, Universite Catholique de Louvain, Louvain-la-Neuve, Belgium, 2003.

[39] A. Mamun and N. Visaltanachoti, "Inflation expectation and asset allocation in the presence of an indexed bond," Working Paper, 2006.

[40] P. Battocchio and F. Menoncin, "Optimal pension management in a stochastic framework," Insurance: Mathematics and Economics, vol. 34, no. 1, pp. 79-95, 2004. 
[41] A. H. Zhang and C.-O. Ewald, "Optimal investment for a pension fund under inflation risk," Mathematical Methods of Operations Research, vol. 71, no. 2, pp. 353-369, 2010.

[42] N.-W. Han and M.-W. Hung, "Optimal asset allocation for DC pension plans under inflation," Insurance: Mathematics and Economics, vol. 51, no. 1, pp. 172-181, 2012.

[43] P. Battocchio and F. Menoncin, "Optimal portfolio strategies with stochastic wage income and inflation: the case of a defined contribution pension plan," Working Paper, 2002.

[44] A. Zhang, R. Korn, and C.-O. Ewald, "Optimal management and inflation protection for defined contribution pension plans," Blätter der DGVFM, vol. 28, no. 2, pp. 239-258, 2007.

[45] F. de Jong, "Pension fund investments and the valuation of liabilities under conditional indexation," Insurance: Mathematics and Economics, vol. 42, no. 1, pp. 1-13, 2008.

[46] F. Menoncin, "Optimal real consumption and asset allocation for a HARA investor with labour income," Working Paper, 2003, http://ideas.repec.org/p/ctl/louvir/2003015.html.

[47] Y.-Y. Chou, N.-W. Han, and M.-W. Hung, "Optimal portfolioconsumption choice under stochastic inflation with nominal and indexed bonds," Applied Stochastic Models in Business and Industry, vol. 27, no. 6, pp. 691-706, 2011.

[48] A. Paradiso, P. Casadio, and B. B. Rao, "US inflation and consumption: a long-term perspective with a level shift," Economic Modelling, vol. 29, no. 5, pp. 1837-1849, 2012.

[49] R. Korn, T. K. Siu, and A. H. Zhang, "Asset allocation for a DC pension fund under regime switching environment," European Actuarial Journal, vol. 1, supplement 2, pp. S361-S377, 2011.

[50] H. K. Koo, "Consumption and portfolio selection with labor income: a continuous time approach," Mathematical Finance, vol. 8, no. 1, pp. 49-65, 1998.

[51] N. V. Krylov, Controlled Diffusion Processes, vol. 14 of Stochastic Modelling and Applied Probability, Springer, Berlin, Germany, 1980. 


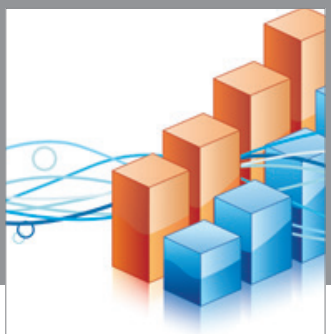

Advances in

Operations Research

vatem alat4

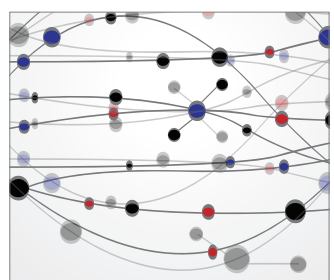

\section{The Scientific} World Journal
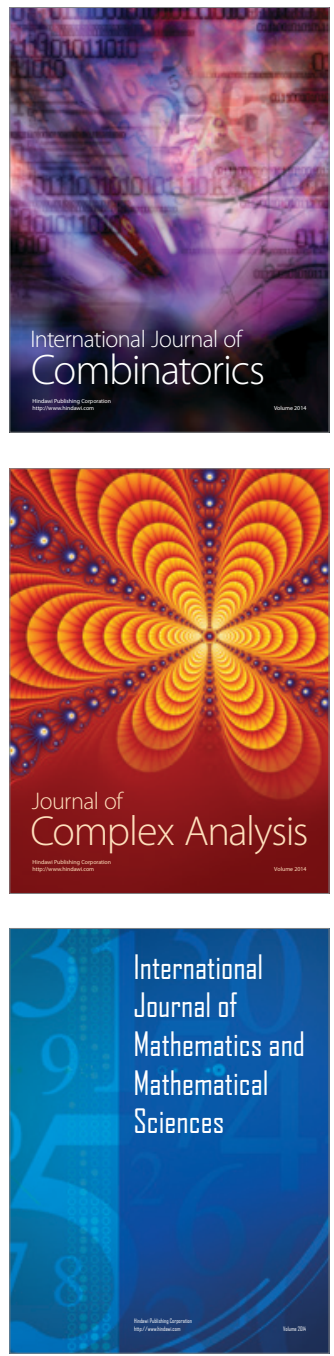
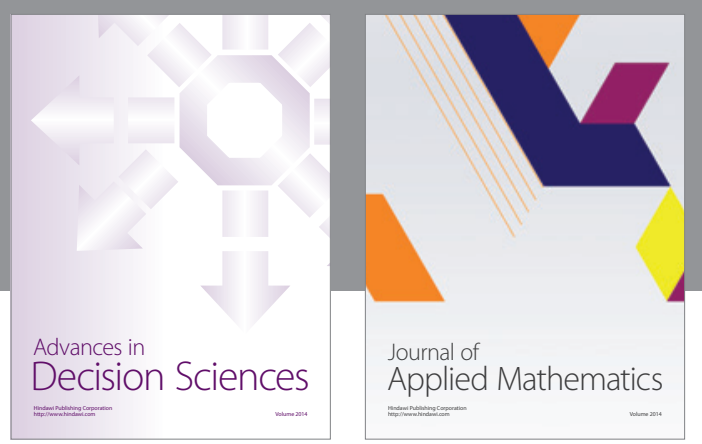

Algebra

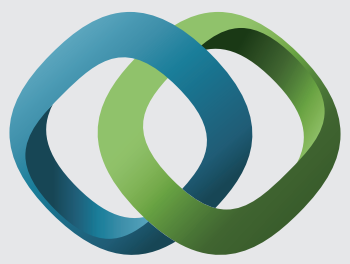

\section{Hindawi}

Submit your manuscripts at

http://www.hindawi.com
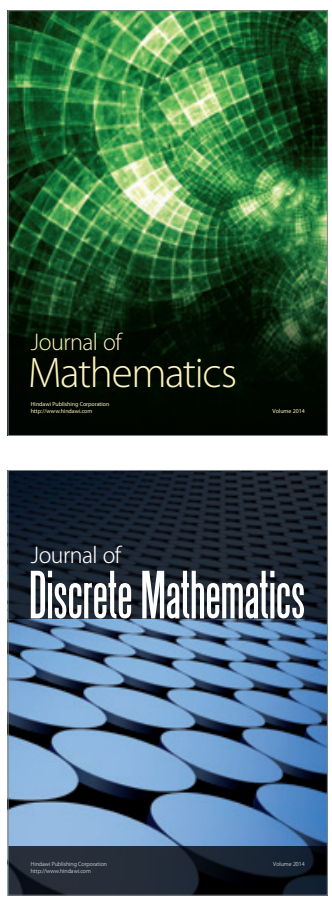

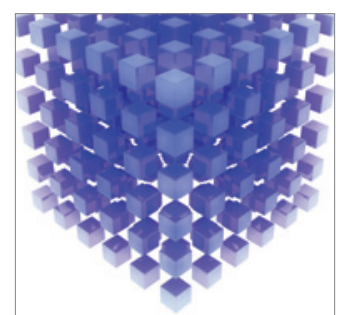

Mathematical Problems in Engineering
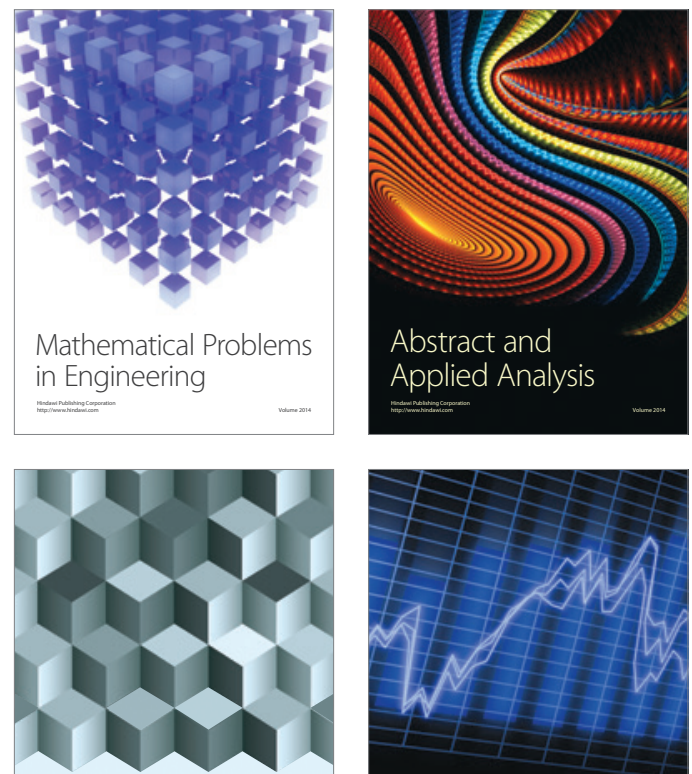

Journal of

Function Spaces

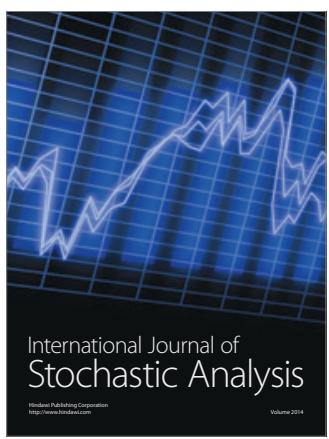

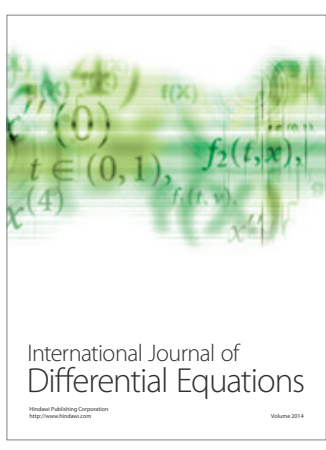
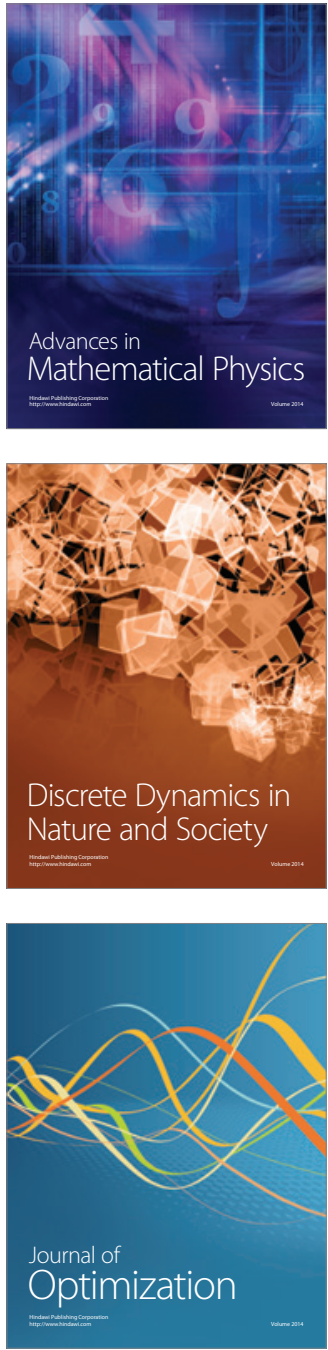